\title{
Effects of a dynamic trailing-edge flap on the aerodynamic performance and flow structures in hovering flight
}

\author{
Chengyu Li, Haibo Dong*, and Geng Liu \\ Department of Mechanical \& Aerospace Engineering \\ University of Virginia, Charlottesville, VA 22904
}

\begin{abstract}
To examine the effects of wing morphing on unsteady aerodynamics, deformable flapping plates are numerically studied in a low-Reynolds-number flow. Simulations are carried out using an in-house immersed-boundary-method-based direct numerical simulation (DNS) solver. In current work, chord-wise camber is modeled by a hinge connecting two rigid components. The leading portion is driven by a biological hovering motion along a horizontal stroke plane. The hinged trailing-edge flap (TEF) is controlled by a prescribed harmonic deflection motion. The effects of TEF deflection amplitude, deflection phase difference, hinge location, and Reynolds number on the aerodynamic performance and flow structures are investigated. The results show that the unsteady aerodynamic performance of deformable flapping plates is dominated by the TEF deflection phase difference, which directly affects the strength of the leading-edge vortex (LEV) and thus influences the entire vortex shedding process. The overall lift enhancement can reach up to $26 \%$ by tailoring the deflection amplitude and deflection phase difference. It is also found that the role of the dynamic TEF played in the flapping flight is consistent over a range of hinge locations and Reynolds numbers. Results from a low aspect-ratio $(A R=2)$ deformable plate show the same trend as those of 2-D cases despite the effect of the three-dimensionality.
\end{abstract}

Keywords: Flapping wing, Hovering flight, Dynamic trailing-edge flap, Aerodynamics, Direct numerical simulation.

\section{Introduction}

Flapping-wing offers unique force-producing mechanisms over conventional flight methods for designing micro air vehicles (MAVs), especially in the low Reynolds number ( $R e$ ) regime. The inherently unsteady nature of flapping kinematics is responsible for the primary force production (Dickinson et al., 1999), and also differentiates flapping-wing motion from conventional fixed- and rotary-wing configurations. In recent years, increasing attention has been paid to the aerodynamics of deformable wings. Several studies have shown that the unsteady aerodynamic phenomena of the flapping mechanism are not only sensitive to variations in the wing kinematics but also to the wing morphing (Valasek, 2012; Wang et al., 2003). The results also reveal that a dynamically adjustable wing surface may potentially provide new aerodynamic mechanisms (Shyy et al., 2011; Zhao et al., 2011) of force production over fully rigid wings (Dickinson et al., 1999; Sun and Tang, 2002) in flapping flight. Further research (Dai et al., 2012; Shoele and Zhu, 2013) has illustrated that the performance of a rigid flapping wing can be significantly improved by adding some level of flexibility to the wing surface. For achieving the performance enhancement, both passive and active flow control mechanisms have been studied, and some examples are reviewed here.

In order to understand the effects of chord-wise flexibility on aerodynamics of flapping motion, a hinge connected two rigid components model is commonly used because of its simplicity and well preservation of the flexibility characteristics in chord-wise (Toomey and Eldredge, 2008). Through this passive deformable model, Eldredge et al. revealed that wings with moderate flexibility have better power

\footnotetext{
*Author to whom correspondence should be addressed: haibo.dong@virginia.edu
} 
efficiency compared to the rigid wing in hovering flight, nevertheless very flexible wings will degrade its aerodynamic performance (Eldredge et al., 2010). Using a similar model, Vanella et al. showed that if parameters are chosen appropriately, the chord-wise flexibility can result in an enhancement of up to $28 \%$ in the lift-to-drag ratio and a 39\% increase in the lift-to-power ratio over a rigid plate (Vanella et al., 2009). Wan et al. studied the effect of chord-wise flexibility over a range of hovering kinematic parameters using a hinged-plate model (Wan et al., 2012). Their results indicate that the maximum lift-todrag ratio can be achieved by placing the hinge at the three-quarter chord position from the leading edge.

In addition to above passive mechanisms explorations, active flow control via wing surface morphing has been widely adopted in both fixed-wing and rotary-wing aircraft design. These include articulated flaps and/or slats (Lee and Su, 2011b), surface flow control devices (Ho and Tai, 1996) and continuously deforming surfaces (Brian C. Prock, 2002). Among them, the flow control via trailing-edge flap (TEF) is presumed to be more applicable to novel flapping-wing micro air vehicles (MAVs) designs, in terms of simplicity of moving surface control, weight of MAVs and so on. Liu et al. experimentally studied the effect of actively controlled trailing-edge flaps on the flow control of translational plates (Liu et al., 2013). Their results have shown that force and flow characteristics strongly depend on the timing of trailing-edge flap deflection rather than translational speed. Li et al. (Li et al., 2014) and Xu et al. (Xu et al., 2015) further studied the effect of the trailing-edge flap on the aerodynamic performance of a 2-D flapping wing via a computational approach. It is found that the aerodynamic forces can be significantly affected by controlling the timing of the flap deflection in a flapping cycle. More extensive reviews of the studies on deformable wings can be found in (Kang et al., 2011; Shyy et al., 2010; Shyy et al., 2008).

However, there is still a lack of effort on quantitative evaluating the possibility of active flow control in flapping-wing MAVs design. To this end, the aerodynamic performance of deformable flapping wings via dynamic trailing-edge flap is deserved to be investigated. In this paper, the unsteady aerodynamics of 2-D and 3-D flapping plates with a controllable trailing-edge flap are computationally studied. Through a systematic parametric study, the effects of TEF deflection amplitude, deflection phase difference, hinge location, and Reynolds number on the near field and far field flow structures and their associated aerodynamic performance are investigated. Both instantaneous and cycle-averaged forces productions are examined to provide quantitative descriptions of the TEF effects. The rest of the paper is organized as follows: Section 2 presents the problem formulation, numerical method, and code validation; Section 3 discusses the simulation results and analysis on aerodynamic performance and vortical structures; and finally, the conclusions of this work are given in Section 4.

\section{Problem definition and numerical approach}

\subsection{Problem Definition}

In the present paper, a thin rigid plate attached with a TEF is considered as the model of deformable flapping wing, as shown in Fig. 1(a). The methodology of handling this thin (0.04 chord-length thickness) plate is demonstrated in (Mittal et al., 2008). As shown in Fig. 1(b), the harmonic kinematics is used to prescribe the flapping motion in a Cartesian coordinates system. Specifically, the main plate is constrained to move in a horizontal stroke plane according to the function defined by Eq. (1) and (2):

$$
\begin{gathered}
x(t)=\frac{A_{0}}{2} \cos (2 \pi f t), \quad y(t)=0 \\
\theta_{L}(t)=\beta_{L} \sin (2 \pi f t)
\end{gathered}
$$

where, $x(t)$ and $y(t)$ denote the position of the leading edge of the chord, $\theta_{L}(t)$ is the orientation of the main plate relative to the vertical direction, $f$ is the flapping frequency, $A_{0}$ and $\beta_{L}$ are the amplitudes of translation and rotation, respectively. 
The deflection angle $\left(\theta_{T}\right)$ of TEF is defined by Eq. (3). In the current study, the deflection phase difference $(\varphi)$ controls the TEF deflection timing, and thus forms a different camber pattern. The choices of these design parameters are in line with other TEF studies on fixed/rotary wing aerodynamics (Gerontakos and Lee, 2008; Lee and Gerontakos, 2006; Lee and Su, 2011a). By changing the deflection phase difference, the plate will either form a positive camber with $\varphi \in\left(-90^{\circ}, 90^{\circ}\right)$, or form a negative camber with $\varphi \in\left(90^{\circ}, 270^{\circ}\right)$ which is presented as the sum of $\left(90^{\circ}, 180^{\circ}\right)$ and $\left(-180^{\circ},-90^{\circ}\right)$ in the following discussions. Fig. 1(c-d) present the typical samples of a positive camber configuration at $\beta_{T}=60^{\circ}, \varphi=60^{\circ}$ and, a negative camber configuration at $\beta_{T}=60^{\circ}, \varphi=-120^{\circ}$, respectively.

$$
\theta_{T}(t)=\beta_{T} \sin (-2 \pi f t+\varphi)
$$

where, $\beta_{T}$ is the amplitude of deflection, and $\varphi$ is the phase difference between the main plate rotation and trailing-edge deflection.

(a)

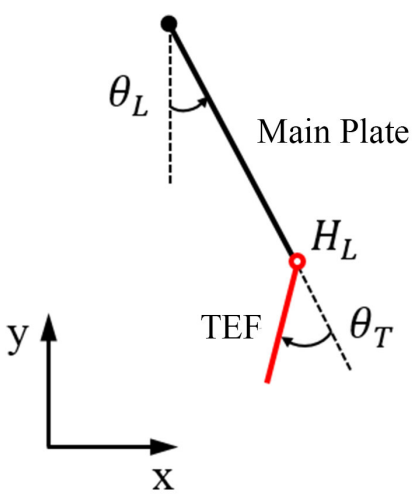

(c)
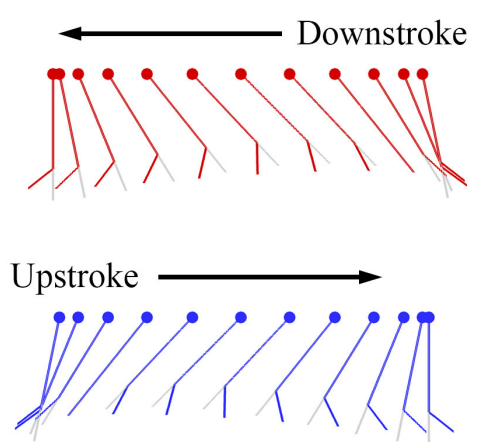

(b)

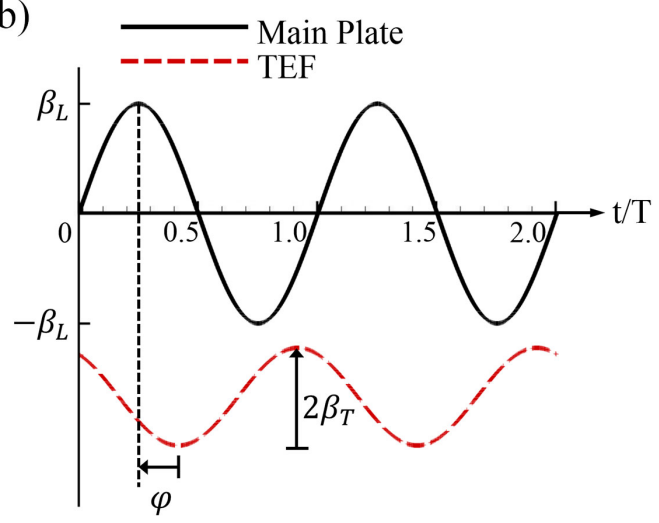

(d)

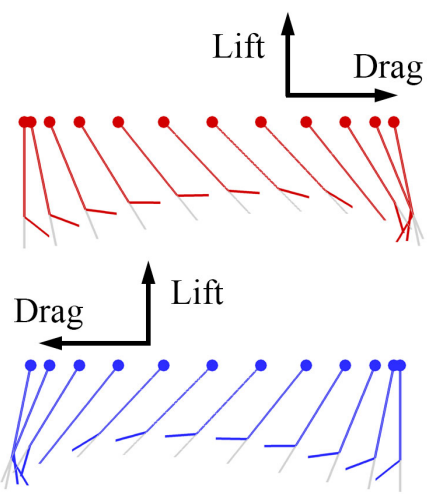

Fig. 1 (a) A schematic illustration of the specified kinematics parameters. The main plate and TEF are colored in black and red, respectively; (b) Definition of flap deflection amplitude $\left(\beta_{T}\right)$ and deflection phase difference $(\varphi)$; (c) Sample of positive camber configuration at $\beta_{T}=60^{\circ}, \varphi=60^{\circ}$; (d) Sample of negative camber configuration at $\beta_{T}=60^{\circ}, \varphi=-120^{\circ}$.

The Reynolds number is defined as $R e=U_{\max } c / v=\pi f A_{0} c / v$, based on the maximum translational velocity ( $\left.U_{\max }\right)$. The lift and drag coefficients are defined as $C_{L}=L /\left(0.5 \rho U_{\max }^{2} c\right)$ and $C_{D}=D /\left(0.5 \rho U_{\max }^{2} c\right)$, with the lift and drag denoted by $L$ and $D$, respectively. 
1 The cycle-averaged lift and drag coefficients are denoted by $\bar{C}_{L}$ and $\bar{C}_{D}$, respectively. The instantaneous 2 aerodynamic power is calculated as $P=\sum_{i=1}^{n} \bar{F}_{i} \cdot \vec{v}_{i}$, where $n$ is the total number of elements, $\bar{F}_{i}$ is the 3 aerodynamic force acting on each element and $\vec{v}_{i}$ is the corresponding velocity of the element. The power 4 coefficient can be expressed as $C_{P W}=P /\left(0.5 \rho U_{\max }^{3} c^{2}\right)$. For the calculation of cycle-averaged aerodynamic 5 power $\left(\bar{C}_{P W}\right)$, only the positive power is considered. Further, the lift-to-power ratio $\left(\bar{C}_{L} / \bar{C}_{P W}\right)$ is used to 6 evaluate the aerodynamic efficiency. Authors also noticed that the projected chord length of the 7 flapping plate is time-dependent, and the time-averaged chord length, $\langle c\rangle_{T}$, might be another choice 8 for calculating the force coefficients (Wang et al., 2014). It has been found that this may cause slight 9 changes in the force magnitude, however, the overall conclusions of this paper remain unaffected.

\subsection{Numerical Method}

The non-dimensional equations governing the flow in the numerical solver are the time-dependent, viscous incompressible Navier-Stokes equations, written in the indicial form as Eq. (4):

$$
\frac{\partial u_{i}}{\partial x_{i}}=0, \quad \frac{\partial u_{i}}{\partial t}+\frac{\partial\left(u_{i} u_{j}\right)}{\partial x_{j}}=-\frac{\partial p}{\partial x_{i}}+\frac{1}{R e} \frac{\partial}{\partial x_{j}}\left(\frac{\partial u_{i}}{\partial x_{j}}\right)
$$

where $u_{i}$ are the velocity components, $p$ is the pressure, and $R e$ is the Reynolds number.

The above equations are discretized using a cell-centered, collocated arrangement of the primitive variables, and is solved using a finite difference-based Cartesian grid immersed boundary method (Mittal et al., 2008). The equations are integrated with time using the fractional step method. A second- order central difference scheme is employed in space discretization. The Eulerian form of the Navier-Stokes equations is discretized on a Cartesian mesh and boundary conditions on the immersed boundary are imposed through a "ghost-cell" procedure. More details about this method and validation of the code can be found in (Dong et al., 2006; Mittal et al., 2008). 

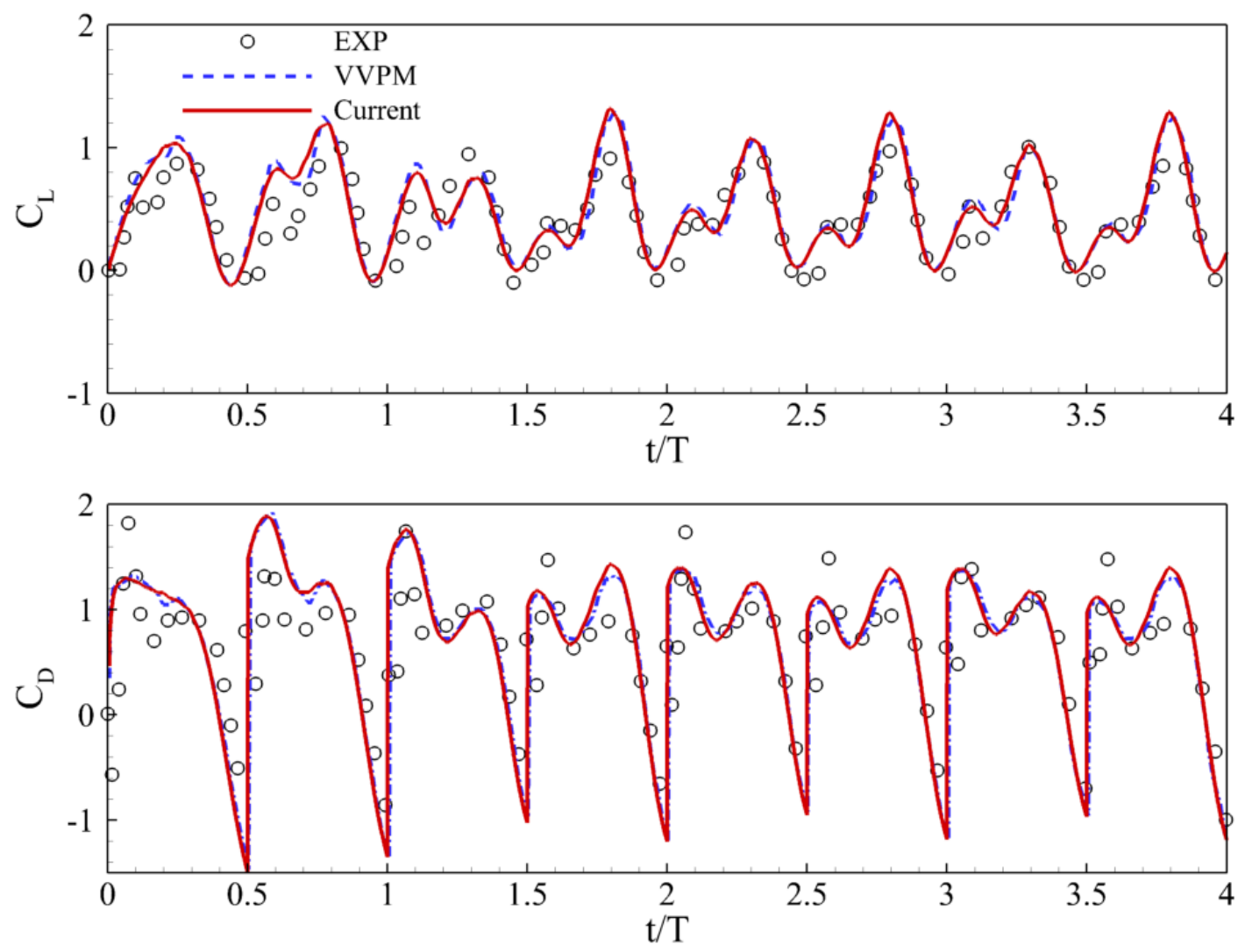

Fig. 2 Lift and drag coefficients from the current simulation, from experimental (EXP) measurement (Wang et al., 2004) and from a viscous vortex particle method (VVPM) simulation (Eldredge, 2007).

\subsection{Validation}

The simulation results obtained for unsteady flow around a thin and rigid wing are compared with previously reported results (Eldredge, 2007; Wang et al., 2004). In this test, the wing rotates around its center, whose orientation is measured counterclockwise relative to the positive $\mathrm{x}$-axis and the amplitude is denoted by $\beta$. The control parameters are otherwise defined the same as in Section 2.1 for the main plate kinematics. To match the simulation setup, $A_{0} / c=2.8$ and $\beta=\pi / 4$ are chosen for the wing kinematics, and the Reynolds number, based on $U_{\max }$ and $c$, is 75 . For the simulation setup, the computational domain size is $30 c \times 40 c$ with a refined zone of $8 c \times 11 c$. The special grid $(321 \times 465)$ is chosen with the smallest resolution of $\Delta x=0.025 \mathrm{c}$ in the dense uniform zone. The flow is initially quiescent. The lift and drag coefficients for the first four cycles are shown in Fig. 2. It can be observed that the present simulation shows good agreement with both experimental measurements (Wang et al., 2004) and numerical results (Eldredge, 2007) obtained using a viscous vortex particle method (VVPM).

In Fig. 3, we present the wake development by showing the vorticity field at different instants. The corresponding flow field from (Eldredge, 2007) is shown for comparison. It can be observed that the instantaneous vortex structures obtained from the two simulations agree with each other very well. 

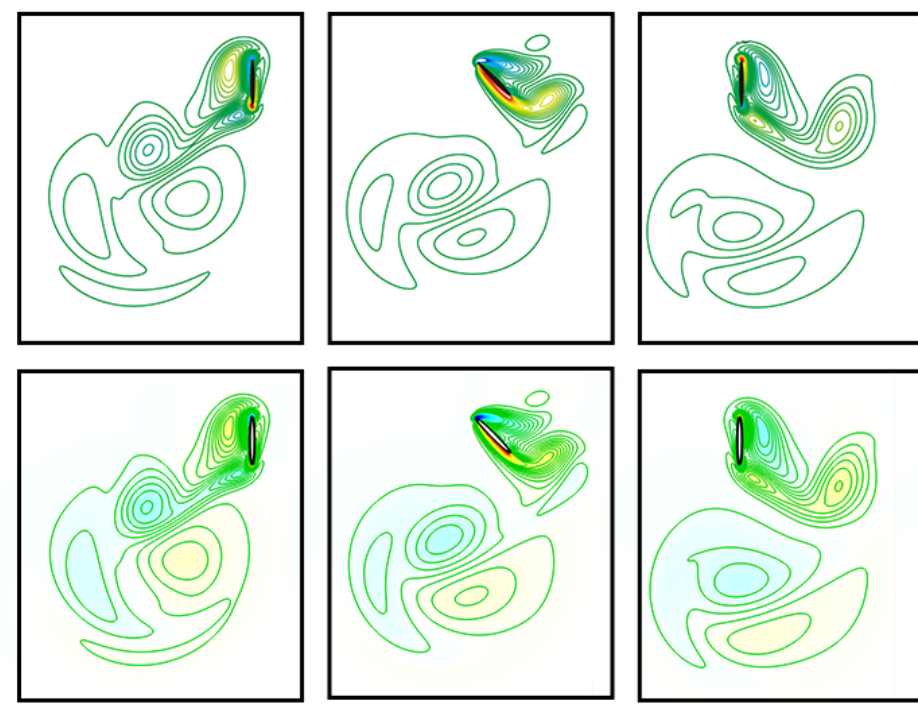

$\mathrm{t} / \mathrm{T}=3$

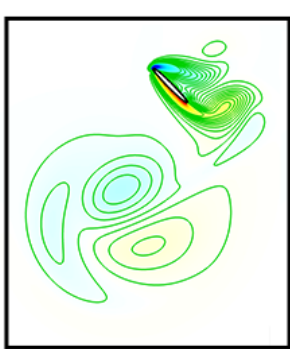

$\mathrm{t} / \mathrm{T}=3.25$

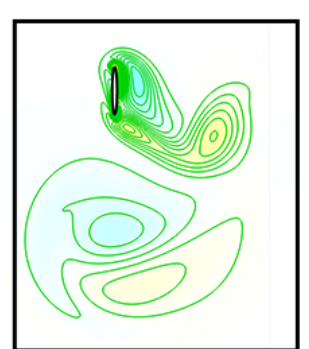

$\mathrm{t} / \mathrm{T}=3.5$
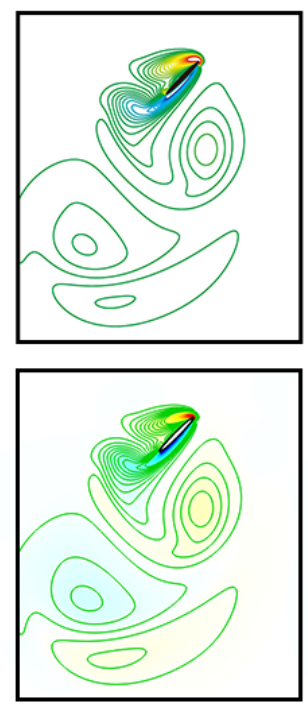

$\mathrm{t} / \mathrm{T}=3.75$
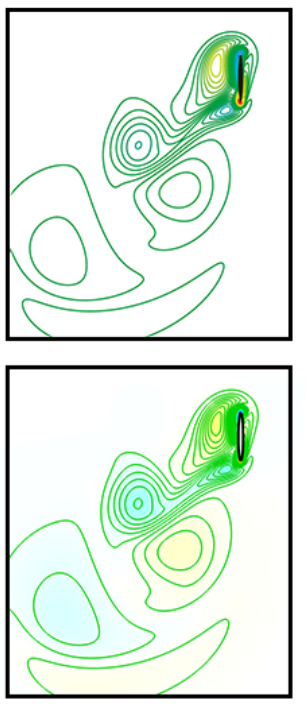

$\mathrm{t} / \mathrm{T}=4.0$

Fig. 3 Vorticity field at the labeled instants with 40 contour levels distributed uniformly between -20 and 20. The top row is from (Eldredge, 2007), and the bottom row is from the current simulation.

\section{Results and discussion}

In this section, we first present the effects of TEF deflection phase difference and deflection amplitude for a hinge location at $H_{L}=0.75 \mathrm{c}$, in Section 3.1 and 3.2, respectively. The effects of the hinge location change will then be investigated in Section 3.3. In these simulations, the following values are selected for the stroke amplitude, the main plate rotational amplitude, and the Reynolds number: $A_{0} / c=3.0$, $\beta_{L}=\pi / 4$, and $R e=100$, respectively. The parameters describing the plate kinematics are chosen based on previous work on insect flight (Eldredge, 2007; Vanella et al., 2009; Wang et al., 2004; Yin and Luo, 2010). The trailing-edge deflection amplitude $\beta_{T}$ ranges from $15^{\circ}$ to $90^{\circ}$, and the deflection phase difference $\varphi$ ranges from $-180^{\circ}$ to $180^{\circ}$. The effects of the Reynolds number will be examined further in Sec. 3.4. Furthermore, low-aspect-ratio 3 -D cases will be analyzed by comparing the aerodynamic performance to the 2-D cases in Section 3.5. The emphasis of the current paper is on the effects of the abovementioned factors on the aerodynamic performance and flow structures.

The computational domain size of 2-D simulation is $30 c \times 40 c$ with a refined zone of $8 c \times 11 c$. The grid $(321 \times 465)$ is chosen with the smallest resolution of $\Delta x=0.02 c$ for the case of $R e=100$, and a finer mesh $(449 \times 673)$ is used for simulations at higher Reynolds number. The 3-D simulations are performed in a $30 c \times 30 c \times 30 c$ domain. The grid adopted has $221 \times 265 \times 117$ cells with minimum grid size $\Delta x=0.03 c$. The time-step ( $T / d t=960)$ is chosen for the current study corresponds to a CourantFriedrichs-Lewy (CFL) number around 0.5. We have conducted extensive tests to ensure that the domain is large enough to achieve accurate results, and grid refinement has been performed to make sure that the simulation results are grid-independent in both the 2-D and 3-D cases. 

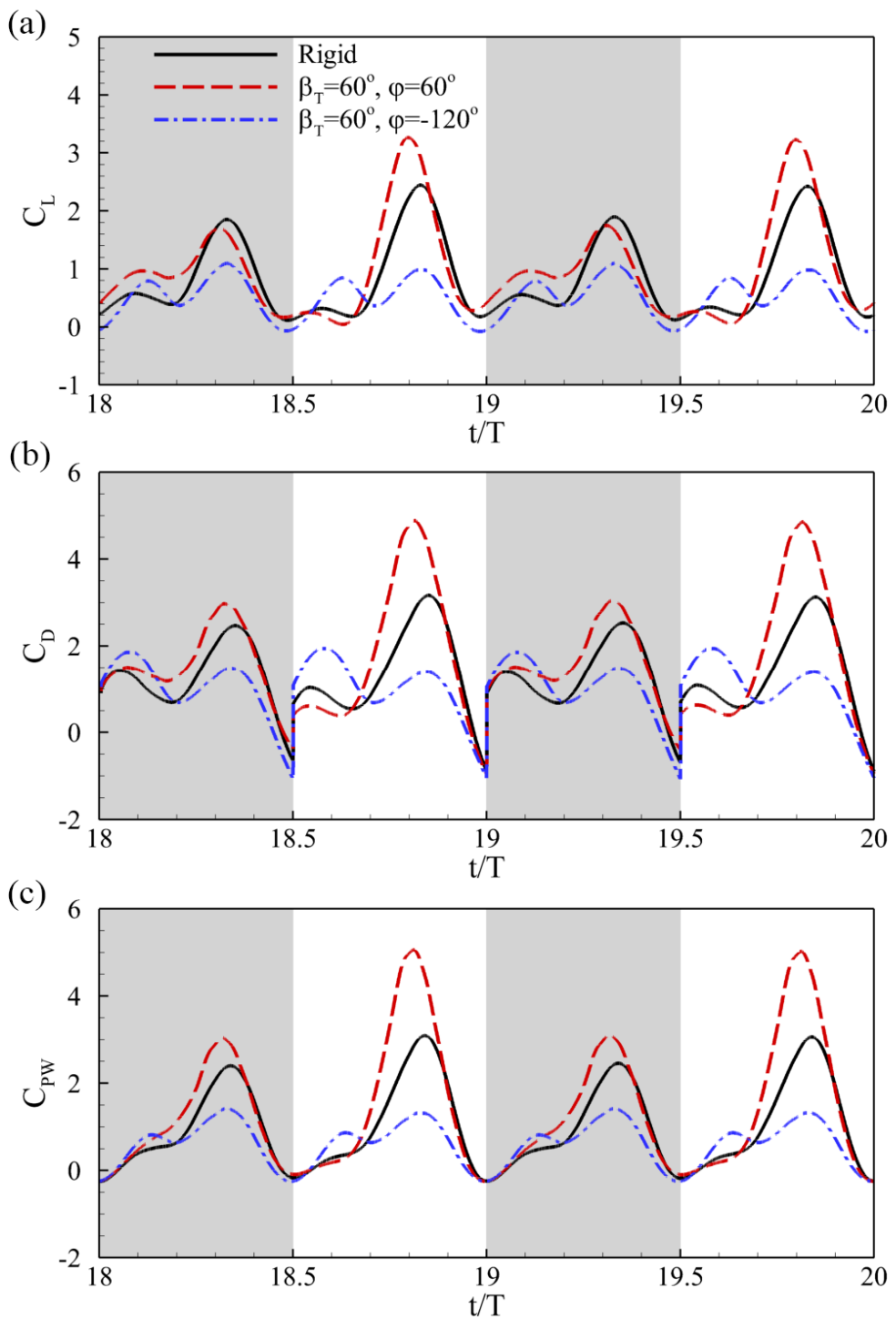

Fig. 4 Time traces of lift (a), drag (b), and power (c) coefficients for fully rigid plate and plate with a TEF at $\beta_{T}=60^{\circ}, \varphi=60^{\circ}$ (positive camber) and $\beta_{T}=60^{\circ}, \varphi=-120^{\circ}$ (negative camber). The half-cycles representing down-stroke are shaded.

\subsection{Effect of TEF deflection phase difference}

To provide a comprehensive picture of how the TEF deflection phase difference affects overall unsteady phenomena, we compare the aerodynamic performance of two typical deformed plate configurations. The two selected cases correspond to positive (Fig. 1(c)) and negative (Fig. 1(d)) camber formations, respectively, with the same deflection amplitude $\left(\beta_{T}=60^{\circ}\right)$. As a reference, data for the corresponding fully rigid plate are also plotted in Fig. 4. Their associated plate configurations are shown in Fig. 1(c-d) for an established cycle.

In general, the instantaneous force history (Fig. 4(a-b)) of all three cases shows two local maxima for each half-cycle. The first peak is associated with the wake capture at the beginning of the stroke, and the second peak is mainly caused by the delayed stall and rotational circulation mechanisms (Dickinson et al., 1999). Between the two peaks is a local minimum referred to as a wake valley, which is caused by a 
combination of decreasing angle-of-attack and interaction with a pocket of downward momentum created during previous flapping cycles (Trizila et al., 2011). The asymmetrical force production between the down- and up-stroke is caused by irregularities in the vortex shedding and wing-vortex interaction (Eldredge et al., 2010).

(a)

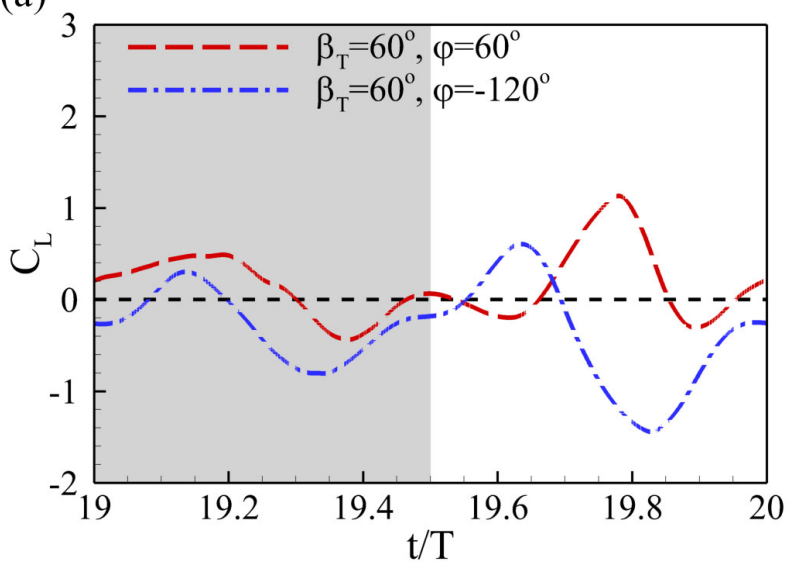

(b)

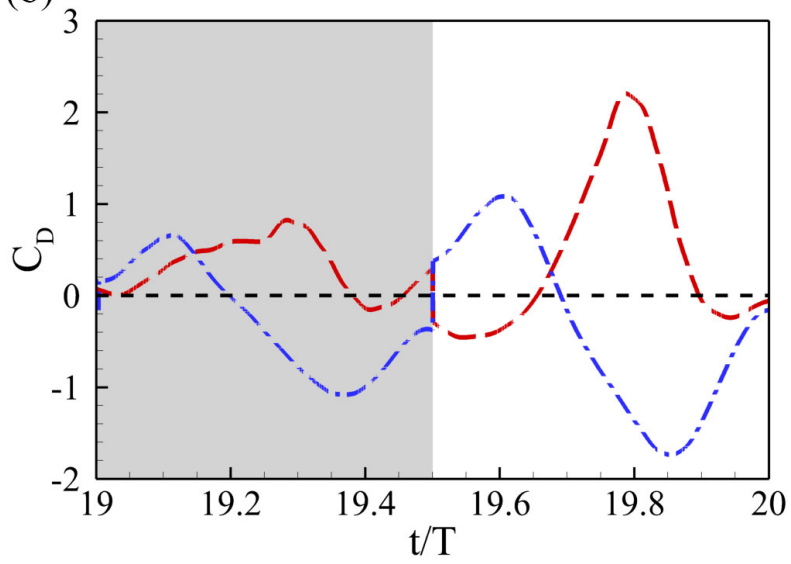

Fig. 5 Time variation of relative force increments compared to the fully rigid plate. (a) $\beta_{T}=60^{\circ}, \varphi=60^{\circ}$. (b) $\beta_{T}=60^{\circ}, \varphi=-120^{\circ}$.

Significant differences can be observed between the case with and without TEF and also between the positive and negative camber formations. By adding TEF deflection motion with different deflection phase difference value, the entire camber formation will be changed. This change will either delay or advance the dynamic stall. As a consequence, the location of the peak values of both forces and power coefficients will be shifted relative to that of the fully rigid case, as can be easily observed in Fig. 4. 


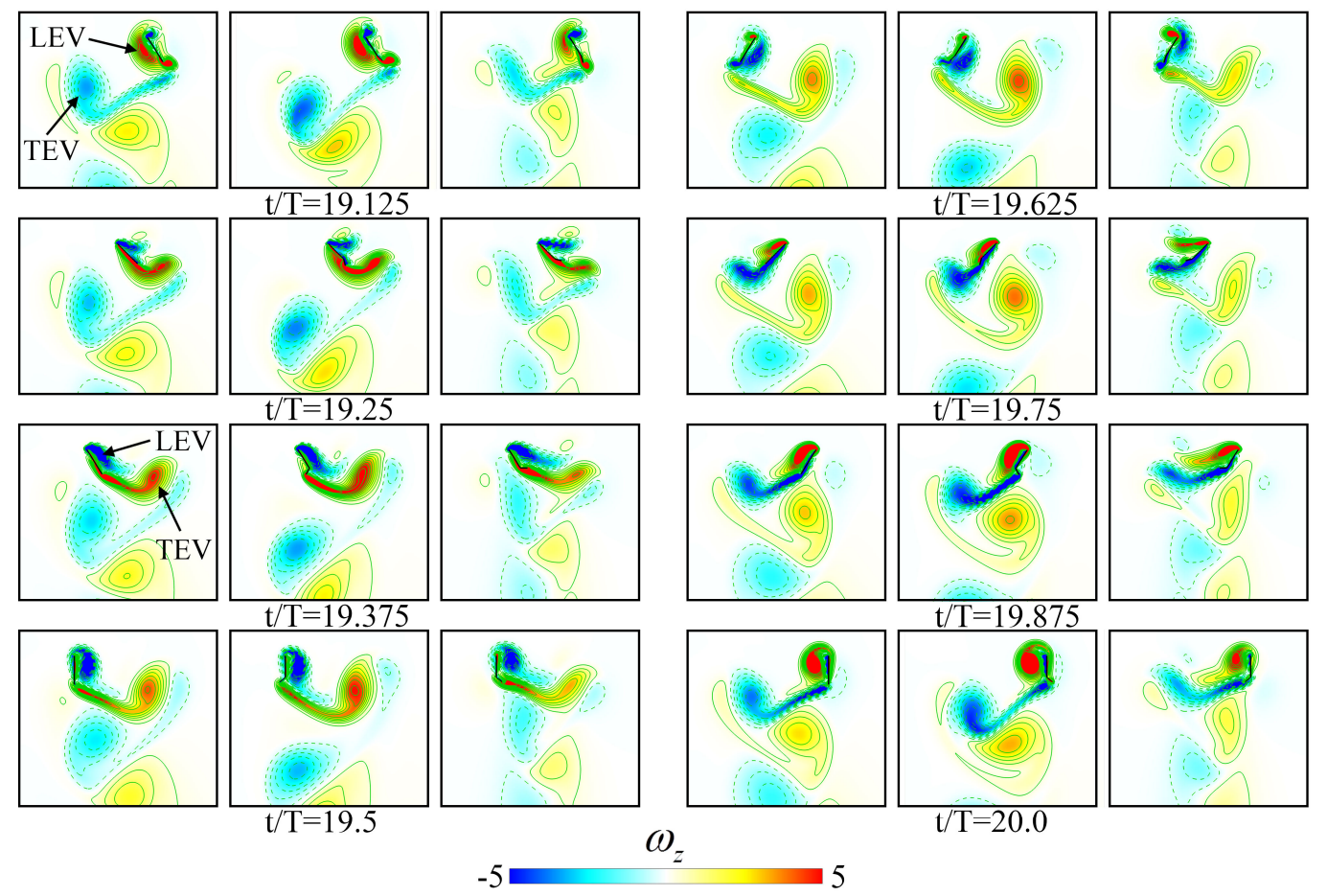

Fig. 6 A series of instantaneous vortex field in a flapping cycle for a fully rigid plate (first and fourth columns), TEF deflection at $\beta_{T}=60^{\circ}, \varphi=60^{\circ}$ (second and fifth columns) and TEF deflection at $\beta_{T}=60^{\circ}, \varphi=-120^{\circ}$ (third and sixth columns). The blue and red colors indicate clockwise and counterclockwise vortices, respectively.

Fig. 4(a) shows that, for the positive camber case, a significant lift enhancement occurs during the upstroke relative to the fully rigid plate, whereas, the negative camber case shows a significant lift decrease. There are two possible reasons for this difference. First, the negative camber formation reduces the frontal area of the deformed plate, as shown in Fig. 1(d). Second, the reverse camber formation prevents the development of leading-edge vortex (LEV). For the latter, the comparison of vortical structure features would alleviate the problem and will be discussed in the following sections. Fig. 4(b) shows that greater drag is produced when the plate experiences higher lift. This result is common for unsteady mechanisms in hovering flight along a horizontal stroke plane. According to the previous literature, an increase in aerodynamic lift due to unsteady effects is typically accompanied by an increase in drag (Dickinson et al., 1999; Wang et al., 2004). The power coefficient displays a similar trend as the drag coefficient varies, as shown in Fig. 4(c). This is because most of the energy has been consumed through drag producing rather than lift producing. For the negative camber case, the power consumption is much lower as a consequence of a lower frontal area.

To better understand the effect of the phase difference of TEF deflection on the force generation, Fig. 5 shows the time course of relative force increments of the plates with two different TEF deflections compared to the fully rigid plate. In which, the relative force increments were obtained by subtracting the forces generated by the fully rigid plate. For the positive camber case ( $\beta_{T}=60^{\circ}, \varphi=60^{\circ}$ ), the maximum force increments happened in the middle of the downstroke and the upstroke. However, the negative camber case $\left(\beta_{T}=60^{\circ}, \varphi=-120^{\circ}\right)$ barely had force increments except during the one-eighth period after the plate reversal, but experienced significant force reduction at the mid-downstroke and the mid-upstroke. 
(a)

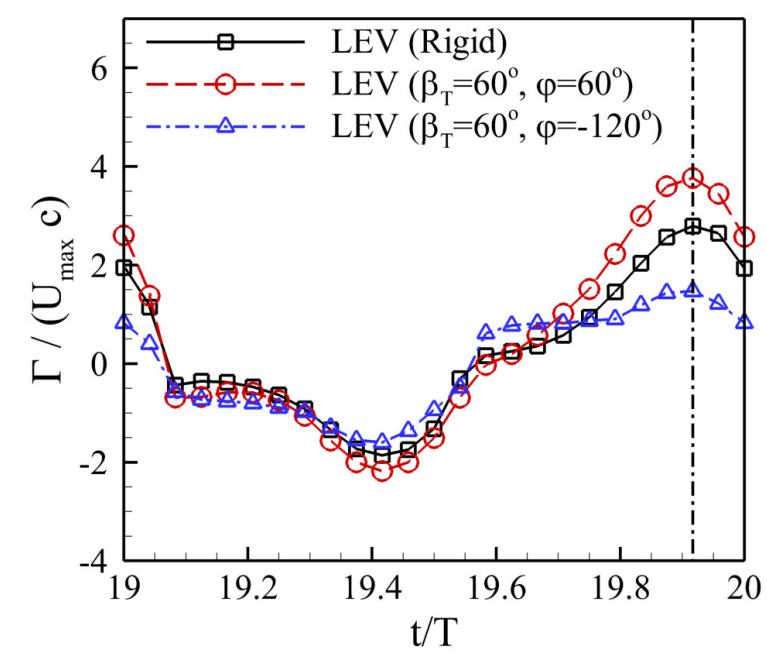

(c)

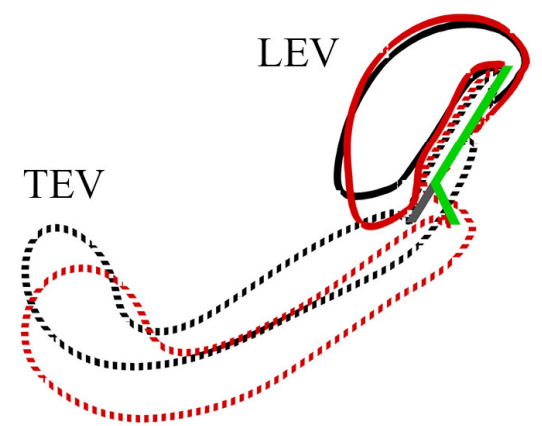

(b)

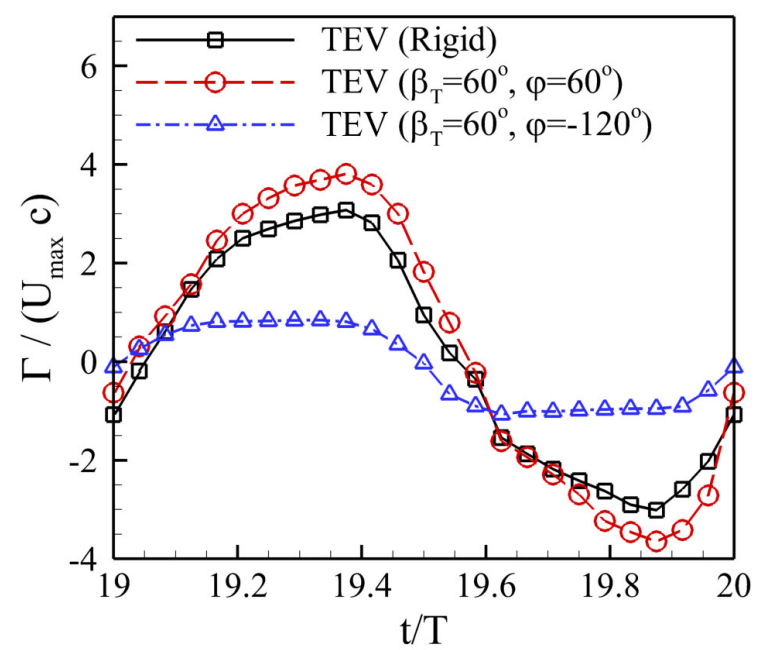

(d)

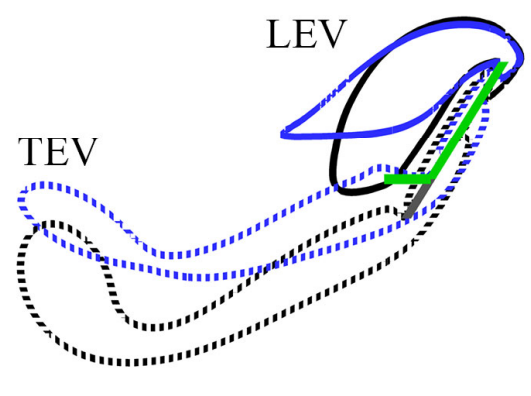

Fig. 7 Comparison of LEV (a) and TEV (b) circulation. The vortex circulation is nondimensionalized by $U_{\max } c$. The positive and negative values correspond to the strength of the vortex during down-stroke and up-stroke, respectively. (c-d) Vortex contour at an instant labeled by the dashed-dot line in (a) for the fully rigid plate (black color), the TEF deflection at $\beta_{T}=60^{\circ}, \varphi=60^{\circ}$ (red color), and the TEF deflection at $\beta_{T}=60^{\circ}, \varphi=-120^{\circ}$ (blue color). The fully rigid and deformed plates are shown as gray and green color, respectively. The solid and dashed line indicates the LEV and TEV, respectively.

To understand the force enhancement mechanism caused by adding a dynamic TEF, we further investigate the near wake structures of all three cases as shown in Fig. 6. The general trend of the vortex formation is similar among all cases. A leading edge vortex (LEV) is generated during each half-stroke and is then recaptured by the plate during its return trip after the stroke reversal. Then the LEV generated by the previous half flapping stroke moves downward along the plate and merges with the current trailing edge vortex (TEV). The merged vortex is strengthened and stretched by the trailing-edge. The wake below the hovering plate is marked by a pair of TEVs with opposite signs that are generated by the two half-strokes in a complete cycle.

The differences of these cases in the flow field are also evident. First, the positive camber case creates a larger size of LEV and TEV than others and its downwash presents a faster downward translational speed. In addition, the dynamic stall can be either advanced or delayed for different camber formations. During the translation phase (as shown in $\mathrm{t} / \mathrm{T}=19.375$ and $\mathrm{t} / \mathrm{T}=19.875$ ), the LEV of the negative camber case presents a clear detachment from the plate suction side. On the contrary, the positive camber case 
shows a better LEV attachment comparing to the fully rigid plate. Furthermore, during the stroke reversal, the positive camber case create a relative stronger wake region over the other cases, which implies the enhancement of the wake capture mechanism (i.e., the negative vortex blob at $t / T=19.5$ and the positive blob at $\mathrm{t} / \mathrm{T}=20.0$ ).

To quantify the strength of the LEV, we further visualize the vorticity field using contour lines. After each vortex is manually identified, a closed contour line is generated around this vortex with a specified level, and the circulation $(\Gamma)$ is then calculated by integrating along this line. Although the magnitude of the circulation depends on the chosen contour level, the characteristic behavior of the vortex is not affected by this choice. The negative and positive signs represent the vortex rotation direction, which can be either clockwise or counterclockwise.

Fig. 7(a-b) shows a comparison of LEV and TEV circulation corresponding to the vortices shown in Fig. 6. In general, the strength and timing of the vortices have significant effects on force production. The peak timing of the TEV circulation appears slightly earlier than that of the LEV circulation. It can be observed that the LEV and TEV for the positive camber case are much stronger than the corresponding vortices for the fully rigid case over a significantly long period. In terms of the flow physics, the wake capture mechanism is enhanced partially due to a stronger flow around the plate at stroke reversal. Note that both enhancement periods appear during the second half of each stroke, when the camber deformation is formed. Unlike that in the positive camber case, the vortex circulation in the negative camber case is much weaker, and the magnitude of LEV circulation is comparable between the down- and up-strokes.

(a)

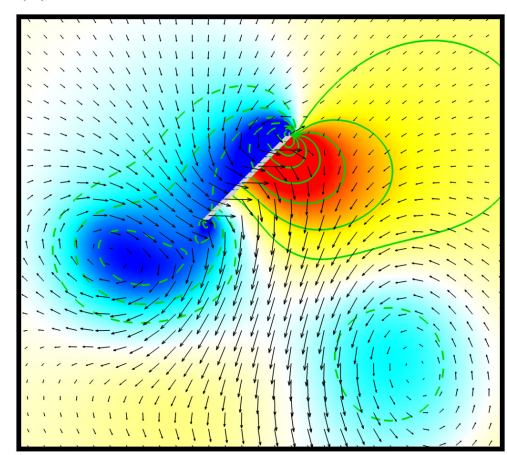

(b)

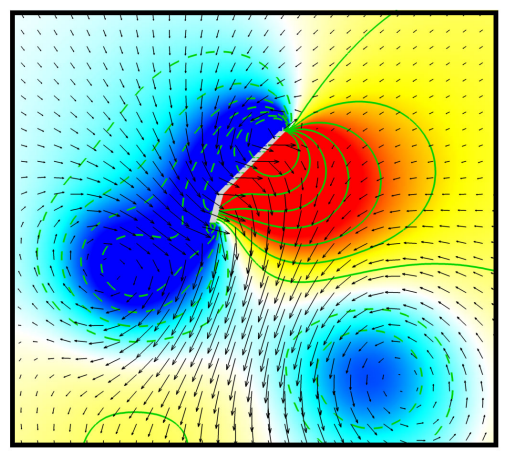

(c)

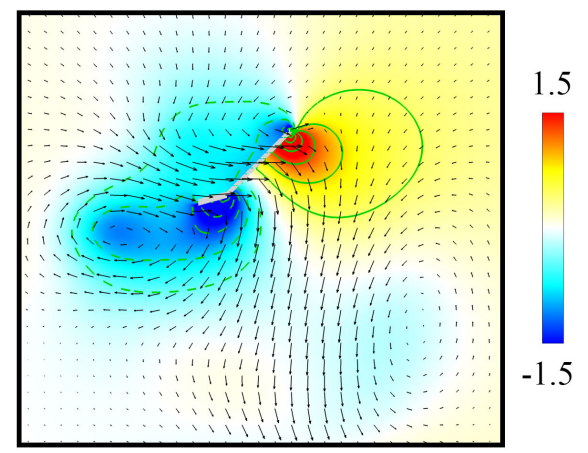

Fig. 8 Comparison of pressure distribution and velocity vector at $t / T=19.75$. (a) Fully rigid plate, (b) Positive cambered plate at $\beta_{T}=60^{\circ}, \varphi=60^{\circ}$, and (c) Negative cambered plate at $\beta_{T}=60^{\circ}, \varphi=-120^{\circ}$. The pressure is normalized by $\rho U_{\max }^{2}$.

Fig. 7(c-d) compares the contour lines of LEV and TEV for an instant that is labeled as a dashed-dot line in Fig. 7(a). Because the magnitude of the circulation is proportional to the area of the contour lines, the difference in circulation can be visualized by comparing the areas of the contour lines. It is observed that the TEF dynamic motion directly stretches its local vortex structure and feeds extra circulation to the TEV (dashed blue line), which results in forming a relatively larger LEV pair. This local flow change also pulls down the LEV structure closer to the plate compared with that observed in the fully rigid case. It is worth noting that the enhancement of LEV and TEV through positive cambering observed in the current work is in line with previous studies on wing flexibilities. These include numerical simulations of insectlike cambering effects of a 2-D wing (Du and Sun, 2008), experimental measurements of robotic rotating wings with varying flexural stiffness (Zhao et al., 2010), and flow-structure interaction of vein-stiffened flapping wings (Shoele and Zhu, 2013). On the other hand, for the negative camber case, the TEF 
stretches its TEV upward. As a consequence, the LEV is pulled up and detached from the suction side of the plate.

The abovementioned local flow modulation will directly alter the pressure distribution. From the normalized pressure contours (Fig. 8), we can see that the positive cambered plate creates a larger lowpressure area on the suction side and the pressure difference between the upper and lower plate surface is clearly increased. The velocity vectors indicate that the positive cambered plate generates a stronger downwash in the flow field comparing to the other two cases. The distribution of the pressure and velocity vector entail the lift generation. Hence, the positive cambered case at this instant experiences a relative larger vertical force, as shown in Fig. 4 at $\mathrm{t} / \mathrm{T}=19.75$. On the opposite, the negative camber case generates a stronger backflow on the suction side and this will pull up the LEV. As a result, the detachment of LEV (dynamic stall) makes the overall force production of the negative camber case drops significantly.

To demonstrate the global impact of the dynamic TEF deflection, Fig. 9 presents the far wake vortical structure. After long-term development, the far-field flow presents a reverse Karman vortex street for all three cases. The positive camber formation results in a more vigorous vortex street (Fig. 9(b)) throughout the entire evolution period. The corresponding overall vortex shedding trajectory presents a slight deflection. It can also be observed that the shedding vortex pair translates faster in the vertical direction. On the other hand, the negative camber case shows an enervated vortex pattern (Fig. 9(c)) with slower translational speed. The shedding vortex gradually vanishes because of the viscosity. In its downwash devolution, the vortex street stays on the same side of the symmetry line of the wake.

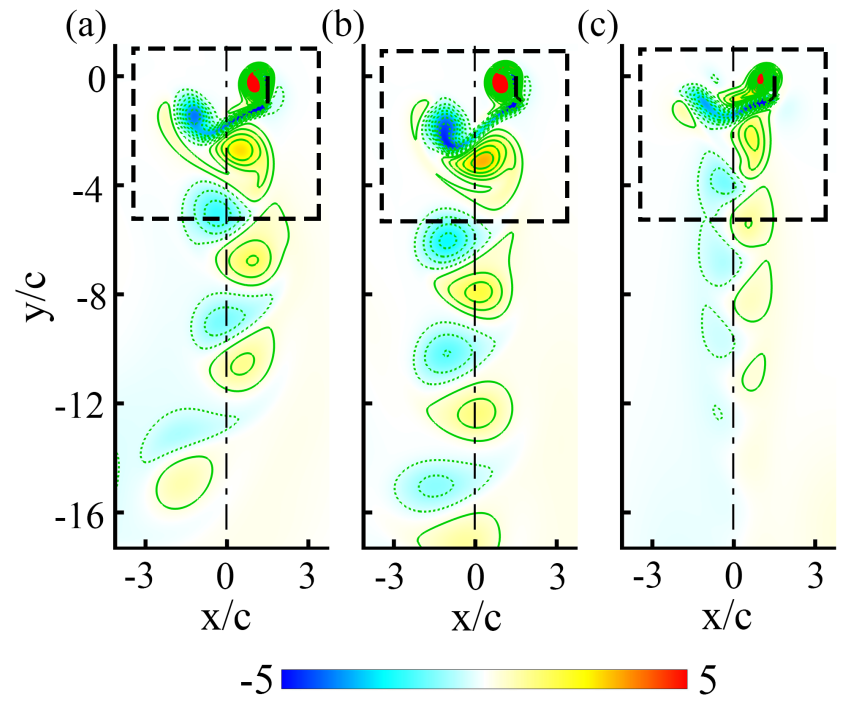

Fig. 9 Far wake vortical structure comparison of fully rigid plate (a), TEF deflection at $\beta_{T}=60^{\circ}, \varphi=60^{\circ}$ (b) and TEF deflection at $\beta_{T}=60^{\circ}, \varphi=-120^{\circ}$ (c) at $\mathrm{t} / \mathrm{T}=20.0$. The dashed square corresponds to the region shown in Fig. 6.

\subsection{Effect of TEF deflection amplitude}

Unlike deflection phase difference effect, adjusting the deflection amplitude will result in a change of camber magnitude. In this section, the effect of deflection amplitude on aerodynamic performance is investigated at $\beta_{T}=15^{\circ}, 30^{\circ}, 60^{\circ}, 90^{\circ}$ for positive camber (at $\varphi=60^{\circ}$ ) and negative camber (at $\varphi=-120^{\circ}$ ) configurations, respectively. The instantaneous forces and power coefficients are plotted in Fig. 10. The 
1 performance of a fully rigid plate is also re-plotted as a reference. The corresponding vorticity fields are 2 shown in Fig. 11.

(a)

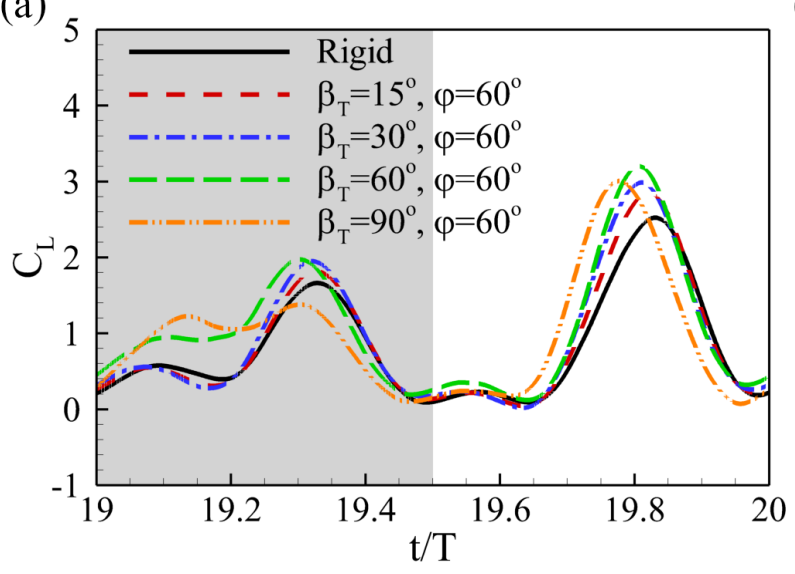

(c)

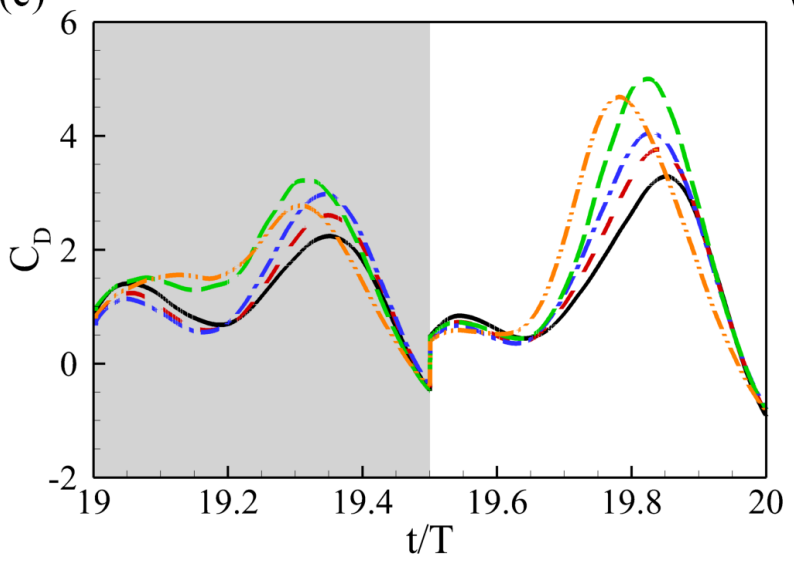

(e)

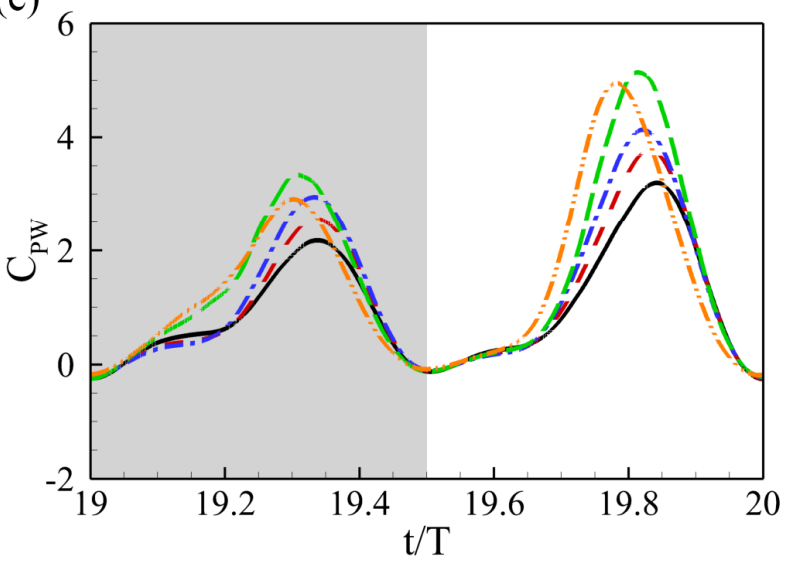

(b)

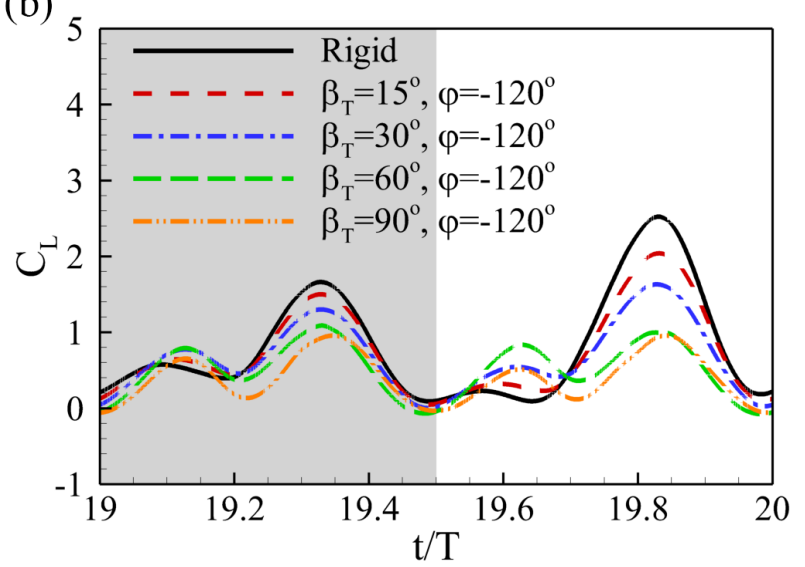

(d)

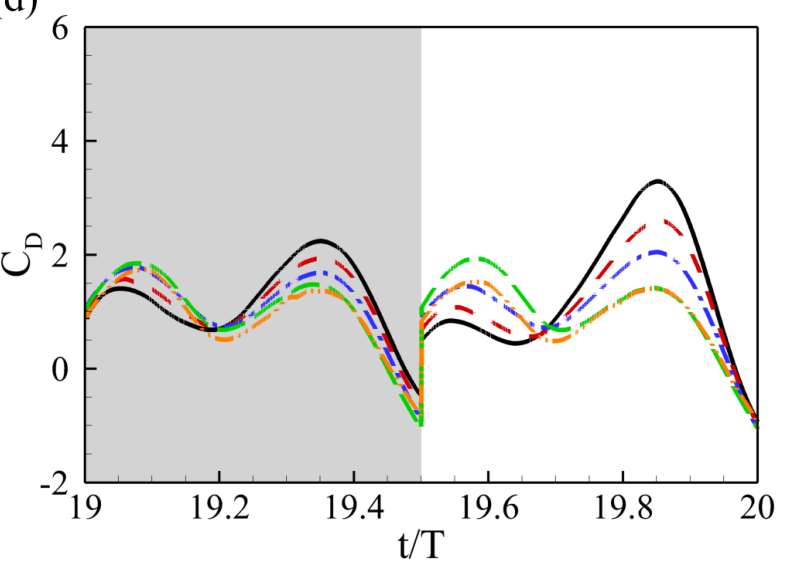

(f)

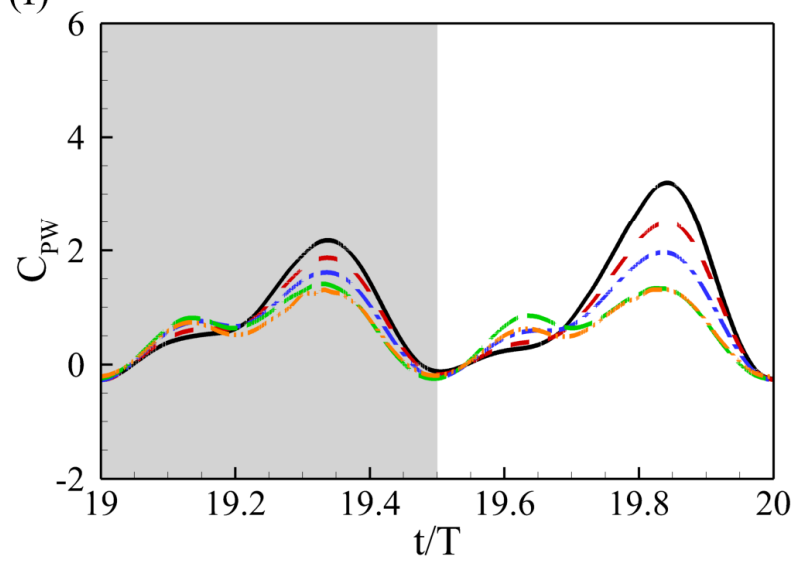

Fig. 10 Time traces of lift (a-b), drag (c-d), and power (e-f) coefficients for positive and negative camber formations at different deflection amplitudes.

In Fig. 10, the first peak of each stroke resulting from the wake capture presents a clear enhancement for the higher deflection amplitude $\left(\beta_{T}=60^{\circ}, 90^{\circ}\right)$ but not for the lower deflection amplitude. The second 
peak of each stroke, which is caused by the delayed stall and rotational circulation mechanisms, shows a gradually increasing trend with deflection amplitude, but it drops when the TEF is over-deflected at $\beta_{T}=90^{\circ}$. For negative camber formation, the first peak increases along with the deflection amplitude, but the second peak presents a decreasing trend at the same time. Although the deflection amplitude change will alter the peak value and appearance timing at certain levels, the overall trend of the instantaneous force and power coefficients for both the positive and negative camber formations are essentially the same. Fig. 11 presents the vortical structures for both the positive and negative camber formations at various deflection amplitudes. The plots show that although the change in deflection amplitude will slightly deflect the vortex street, it will not alter the overall flow pattern dominated by the deflection phase difference.

To systematically explore the effects of TEF on aerodynamic performance, Fig. 12(a-b) shows the lift and drag coefficients averaged over 20 flapping cycles. The results are shown for the TEF deflection amplitudes $\beta_{T}=15^{\circ}, 30^{\circ}, 60^{\circ}, 90^{\circ}$ and deflection phase difference $\varphi$ values ranging from $-180^{\circ}$ to $180^{\circ}$. The performance of a fully rigid plate is indicated by the dash-dot line. It can be observed that for all deflection amplitudes, the TEF deflection phase difference has a significant effect on the force production. Specifically, the optimal lift appears over the range of $\varphi=\left(0^{\circ}, 90^{\circ}\right)$, and the peak value is up to $26 \%$ higher than that of a fully rigid plate. The deflection phase difference range $\varphi=\left(0^{\circ}, 90^{\circ}\right)$ also leads to an increase of at least $40 \%$ in the drag coefficient compared with that of the fully rigid plate. Although drag production is not desirable for the steady motion, it has the potential to enhance maneuverability if an asymmetric deflection phase difference value is selected between the inner and outer flapping-wings because the torque generation for inset flight is mainly drag-based (Bergou et al., 2010). For variations in the deflection phase difference over the ranges $\left(-180^{\circ},-90^{\circ}\right)$ and $\left(90^{\circ}, 180^{\circ}\right)$, both the lift and drag decrease, especially for the larger deflection amplitude cases. This decrease is observed due to the decrease in the frontal area when a reverse camber is formed for $\varphi$ over these ranges.

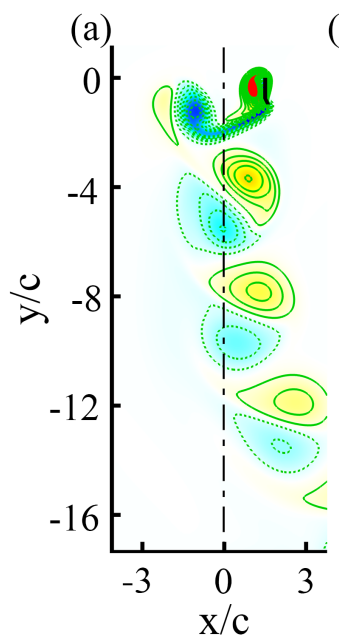

(b)

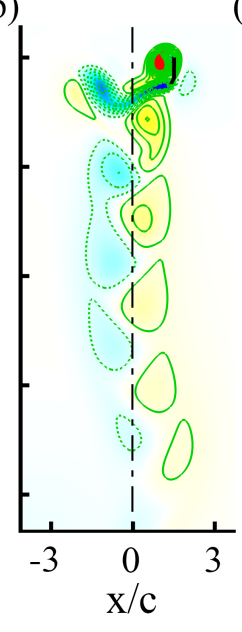

(c)

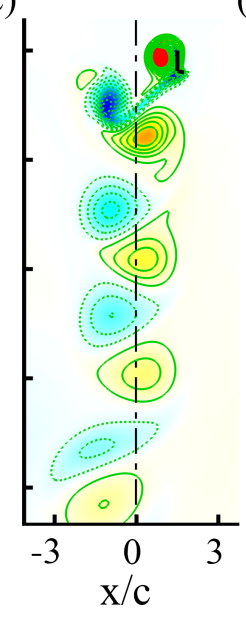

(d)

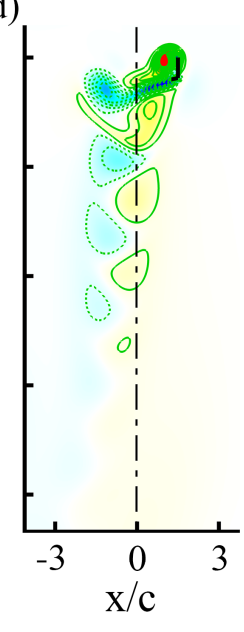

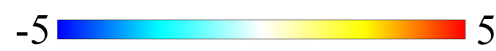

5

Fig. 11 Far wake vortical structure comparison of TEF deflection at $\beta_{T}=30^{\circ}, \varphi=60^{\circ}$ (a), $\beta_{T}=30^{\circ}, \varphi=-120^{\circ}(\mathrm{b}), \beta_{T}=90^{\circ}, \varphi=60^{\circ}$ (c), and $\beta_{T}=90^{\circ}, \varphi=-120^{\circ}$ (d).

In Fig. 12(c), the power calculation is based on the input from the leading edge of the plate, and negative power is not taken into account. Overall, the power coefficient displays a trend similar to that of the drag coefficient. This is again because most of the energy is consumed through drag rather than 
through lift. Unlike the force and power coefficient maps, in which higher values always appear at higher deflection amplitudes, the lift-to-power ratio shown in Fig. 12(d) presents a higher value at lower deflection amplitudes, especially for a negative camber formation. The optimal lift-to-power ratio can reach up to 0.85 , which is $21 \%$ higher than that for the fully rigid plate. The possible reason is that although the negative camber cases own lower lift production, the symmetric flow pattern (i.e., Fig. 9(c)) reduces the power consumption in the horizontal direction. This leads to a higher aerodynamic efficiency in terms of the lift-to-power ratio.

(a)

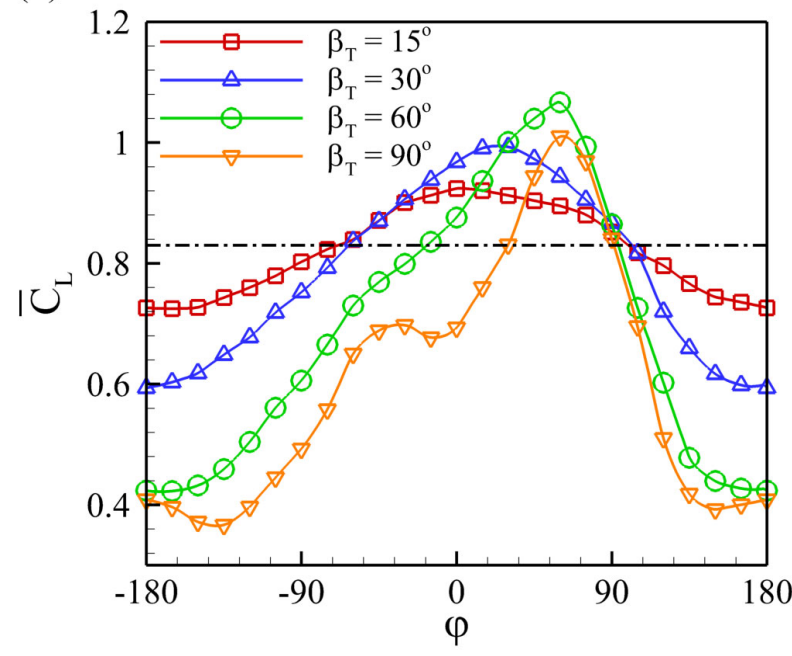

(c)

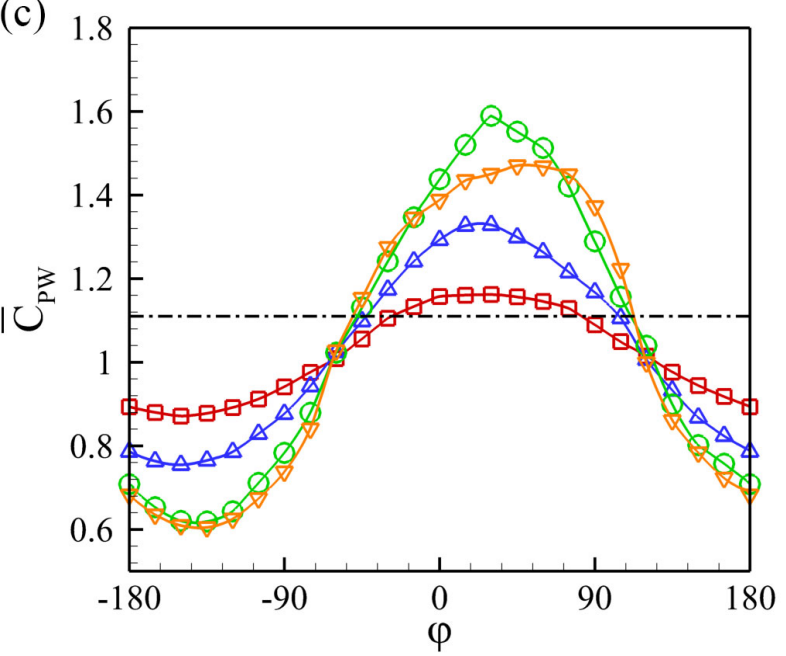

(b)

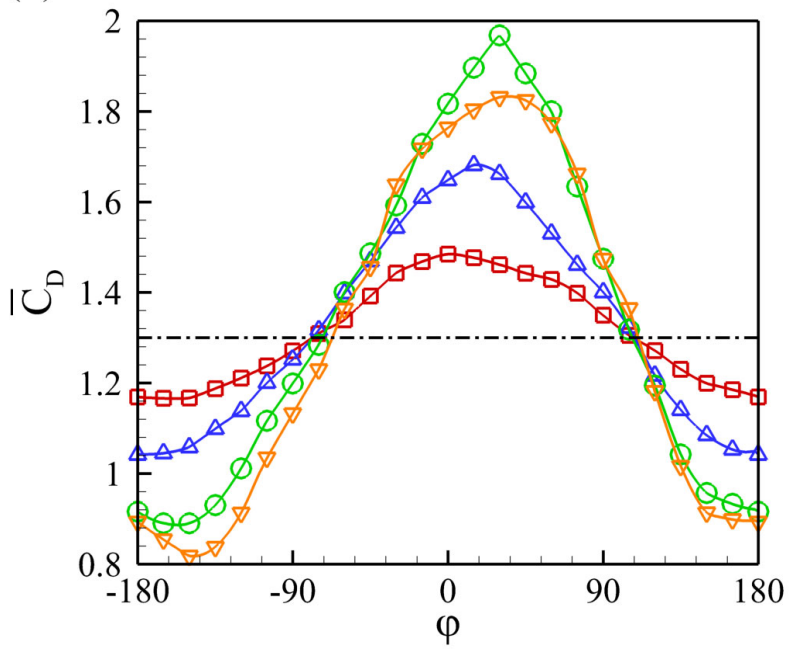

(d)

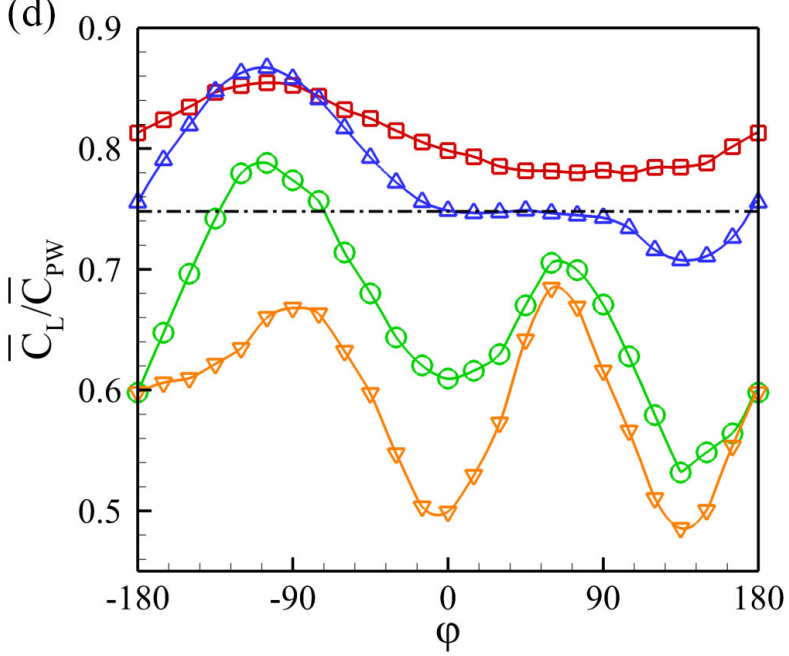

Fig. 12 Aerodynamic performance of plate at $H_{L}=0.75 \mathrm{c}$. Cycle-averaged lift (a), drag (b), and power (c) coefficients, and lift-to-power ratio (d).

Comparing the four deflection amplitudes, we find that there is an optimal value for the magnitude of camber formation. For lift production, a moderate deflection amplitude $\left(\beta_{T}=60^{\circ}\right)$ with positive camber formation generates the optimal lift along with the decrease of the lift-to-power ratio. However, the low deflection amplitude $\left(\beta_{T}=15^{\circ}\right)$ with a positive camber is able to improve both the lift and lift-to-power ratio, but at much smaller level than that of $\beta_{T}=60^{\circ}$. It should be noted that the camber formation at 
$\beta_{T}=15^{\circ}$ corresponds to a maximum $8 \%$ camber-to-chord ratio, where the camber height is defined as the distance between the hinge locations to the straight line connecting leading to trailing edge. This value is close to that observed in real insect wings (Koehler et al., 2012).

Moreover, as shown in Fig. 12, the phase difference of the TEF deflection is another important parameter for controlling the plate's aerodynamic performance. The change of this parameter during flapping is equivalent to the time variation of the camber formation, as shown in Fig. 1(b). Results have shown that the optimal lift production always occurs when the deflection phase difference is within $0^{\circ}<\varphi<90^{\circ}$ (Fig. 12(a)). Meanwhile, the maximum camber is formed when the wing is about to reversal. This is consistent with previous findings in real insect flights. Table 1 shows the comparison of camber formation including camber-to-chord ratio $\left(h_{\max } / c\right)$ and formation timing $\left(\tau_{h \max }\right)$ of the maximum camber between the optimal lift case from the current work and previous studies on real insects. Here, $h_{\max }$ and $c$ represent the maximum camber height and wing chord length, respectively, and $\tau_{h \max }$ is the formation timing of the maximum camber which is normalized by the downstroke duration $\left(T_{\text {down }}\right)$. Results have shown that for various real insects, the maximum camber-to-chord ratios are between 5\% $16 \%$, and they are all formed at the end of the downstroke, $0.7 T_{\text {down }} \sim 0.9 T_{\text {down }}$. Similar trends can also be found from the results of the optimal lift case in the current work despite that the TEF motion is prescribed.

Table 1: The maximum camber-to-chord ratio $\left(h_{\max } / c\right)$ and the timing of maximum camber $\left(\tau_{h \max }\right)$ during downstroke for real insects along with the optimal lift case from the current study.

\begin{tabular}{cccc}
\hline \hline & Flight conditions & $h_{\max } / c$ & $\tau_{h \max }$ \\
\hline $\begin{array}{c}\text { Dragonfly hindwing } \\
\text { (Koehler et al., 2012) }\end{array}$ & Free flight & $5 \%-9 \%$ & 0.73 \\
$\begin{array}{c}\text { Desert locust forewing } \\
\text { (Walker et al., 2009) } \\
\begin{array}{c}\text { Desert locust hindwing } \\
\text { (Walker et al., 2009) } \\
\text { Hoverfly }\end{array}\end{array}$ & Tethered flight & $2 \%-9 \%$ & 0.68 \\
$\begin{array}{c}\text { (Walker et al., 2010) } \\
\text { Butterfly }\end{array}$ & Tethered flight & $5 \%-10 \%$ & 0.72 \\
$\begin{array}{c}\text { (Zheng et al., 2013) } \\
\text { Beetle hindwing } \\
(\text { Le et al., 2013) } \\
\text { Honeybee }\end{array}$ & Forward flight & $6 \%-11 \%$ & 0.71 \\
$\begin{array}{c}\text { (Zhang et al., 2008) } \\
\text { Current work }\end{array}$ & Free flight & $11 \%-16 \%$ & 0.78 \\
$\left(\beta_{T}=60^{\circ}, \varphi=60^{\circ}\right)$ & Free flight & $5 \%-12 \%$ & 0.90 \\
\hline \hline
\end{tabular}

It is worth noting that the insect wings are made of complicated biomaterials coupled with ingenious wing structural designs that are able to extract maximum advantage from elastic tailoring and other mechanisms during the flapping motion. Current work has indicated that by carefully choosing the control parameters, active control of wing surface morphing may be able to achieve biological levels of aerodynamic performance as insect flapping wings. This observation can be treated as a promising inspiration for the wing design of flapping-winged MAVs. 


\subsection{Effect of hinge location}

In previous sections, the discussion is focused on a flapping plate with a hinge located at three-quarter chord length $\left(H_{L}=0.75\right)$ from the leading edge. In this section, the effects of varying hinge location on aerodynamic performance are investigated. For making a fair comparison, we keep the same effective pitching angle for different hinge location cases. The effective pitching angle (as shown in Fig. 13) is defined in the same way as that used in (Dai et al., 2012). For different hinge location cases, the total length of the whole plate is still maintained as unit one.

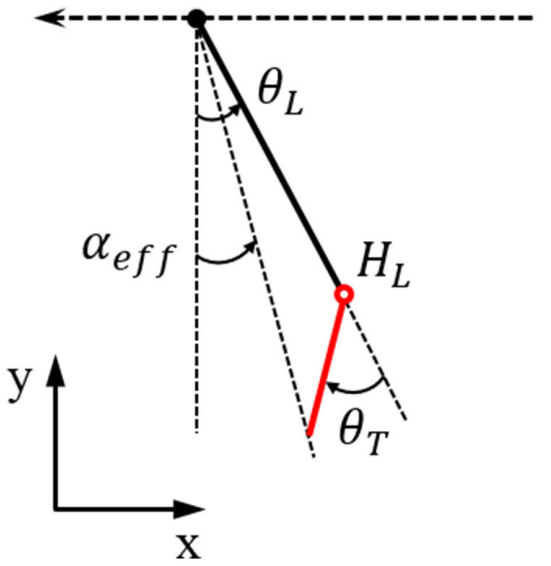

Fig. 13 Definition of effective pitching angle $\left(\alpha_{\text {eff }}\right)$.

Fig. 14 shows the mean aerodynamic performance for different hinge locations. By keeping the same effective pitching angle, the deflection amplitude for varying hinge location is different. For instances, the deflection amplitude $\left(\beta_{T}\right)$ of hinge location at 0.25 , and 0.5 chord length from the leading edge is around $18.5^{\circ}$ and $27.8^{\circ}$, respectively, for achieving the same effective pitching angle as hinge placed at three-quarter chord length cases at $\beta_{T}=60^{\circ}$. In general, the aerodynamic performance follows the same trend for varying hinge locations. However, there still exists slight difference especially for the lift generation. It is demonstrated that changing hinge locations have a different impact on the aerodynamics of positive and negative camber formations. Fig. 14(a) shows that for positive camber, $H_{L}=0.75$ yields the highest lift, whereas for negative camber cases, $H_{L}=0.25$ leads to the greatest lift. More specifically, the averaged lift enhancement of positive camber cases at $H_{L}=0.75$ is $7.2 \%$

higher than the same case at $H_{L}=0.25$. In contrast, the averaged lift for the negative camber formation at $H_{L}=0.25$ is $9 \%$ higher than the same case at $H_{L}=0.75$. In addition, the drag and power coefficients in Fig. 14(b-c) only show slight differences between various hinge locations because the effective angle of attack for all simulations is kept the same. Moreover, the difference in the lift-to-power ratio (as shown in Fig. 14(d)) is directly determined by the change in lift production. 
(a)

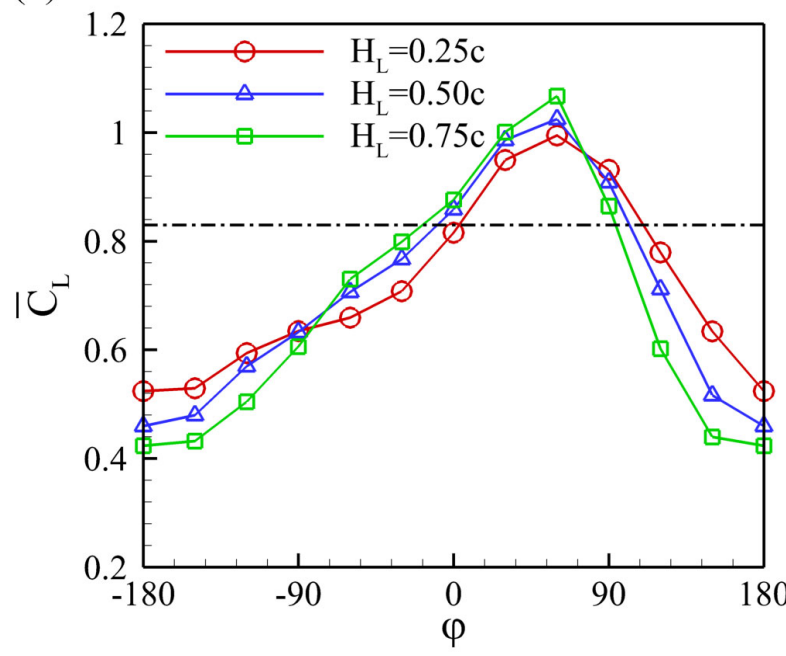

(c)

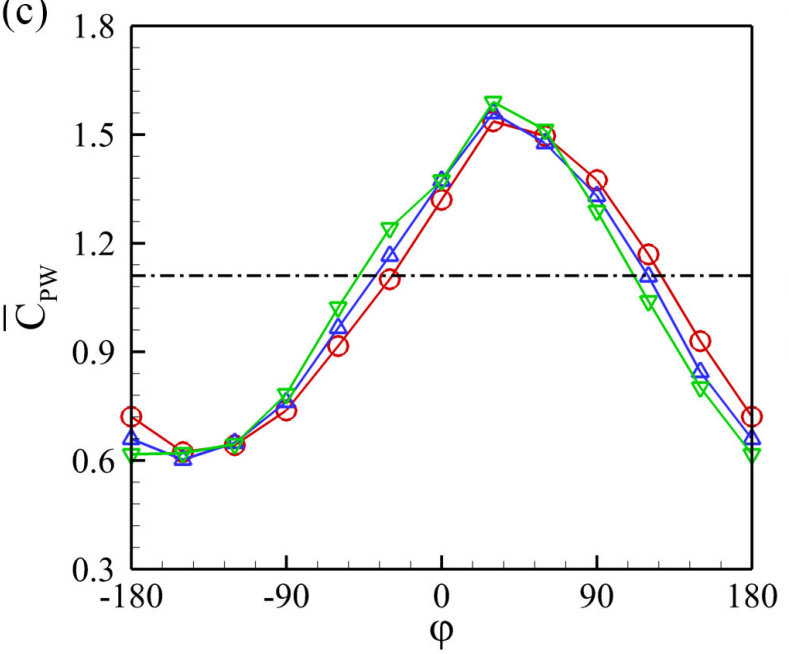

(b)

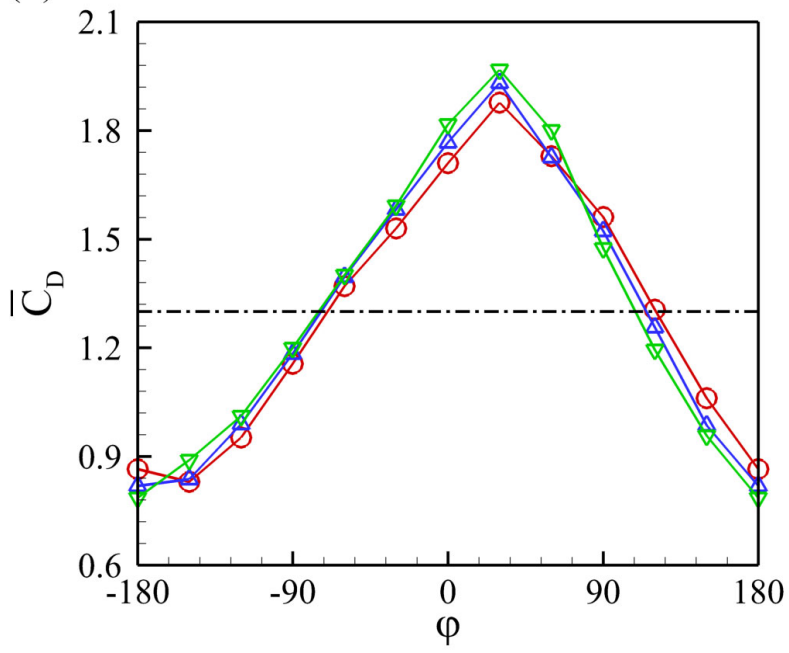

(d)

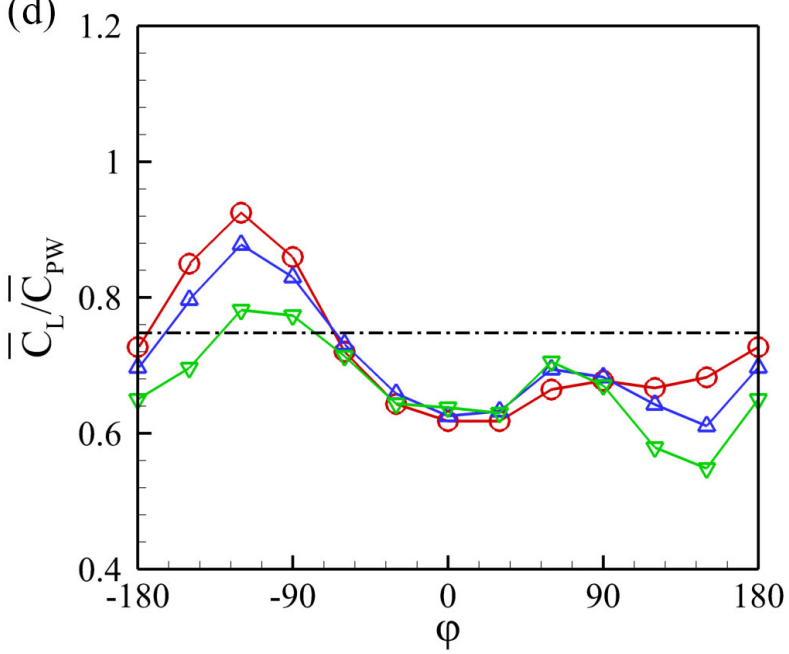

Fig. 14 Aerodynamic performance of plate has different hinge locations. Cycle-averaged lift (a), drag (b), and power (c) coefficients, and lift-to-power ratio (d).

\subsection{Effect of Reynolds number}

In order to study the Reynolds number ( $R e$ ) effects, we have changed $R e$ from 50 to 400 and run the higher Reynolds number cases on finer meshes. At each Reynolds number, simulations are performed at $H_{L}=0.75 \mathrm{c}$ and $\beta_{T}=60^{\circ}$ for different deflection phase difference.

Fig. 15(a-c) show comparisons of the cycle-averaged forces and power coefficients at various $R e$. In general, the lift coefficient increases with Reynolds number, especially for positive camber formation. However, the drag and power coefficients present different trends for positive and negative camber cases respectively when Reynolds number increases. For positive camber cases, both the drag and power coefficients increase with Reynolds number, whereas both the drag and power coefficients decrease as Re increases for negative camber formation. In Fig. 15(d), the lift-to-power ratio always shows an increasing trend with the Reynolds number for different deflection phase difference values. This trend reveals that with the increase in Reynolds number, the dynamic TEF gains more benefit from the unsteadiness of the sounding flow. Although there is a difference in magnitude for the cases with different 
1 Reynolds numbers, the overall performance trend for varying deflection phase difference remains the 2 same.

(a)

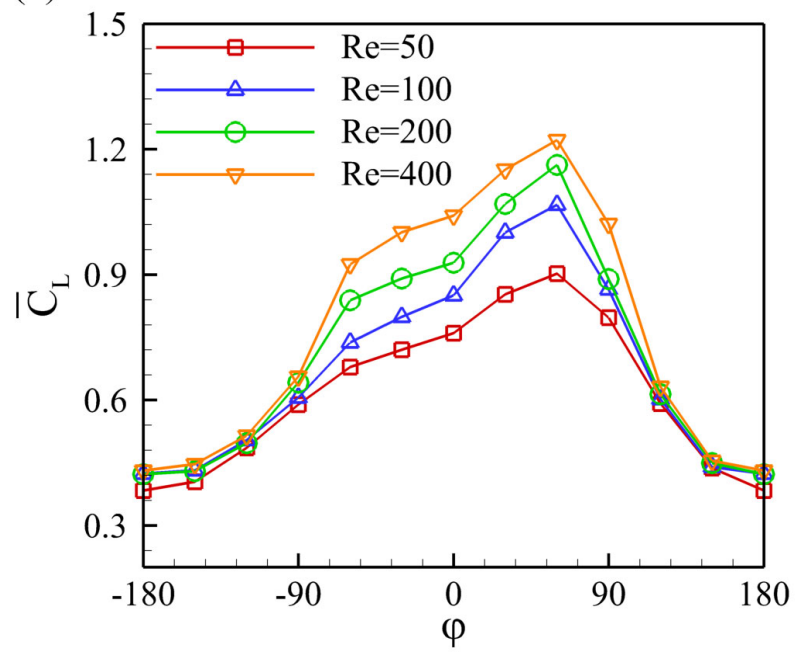

(c)

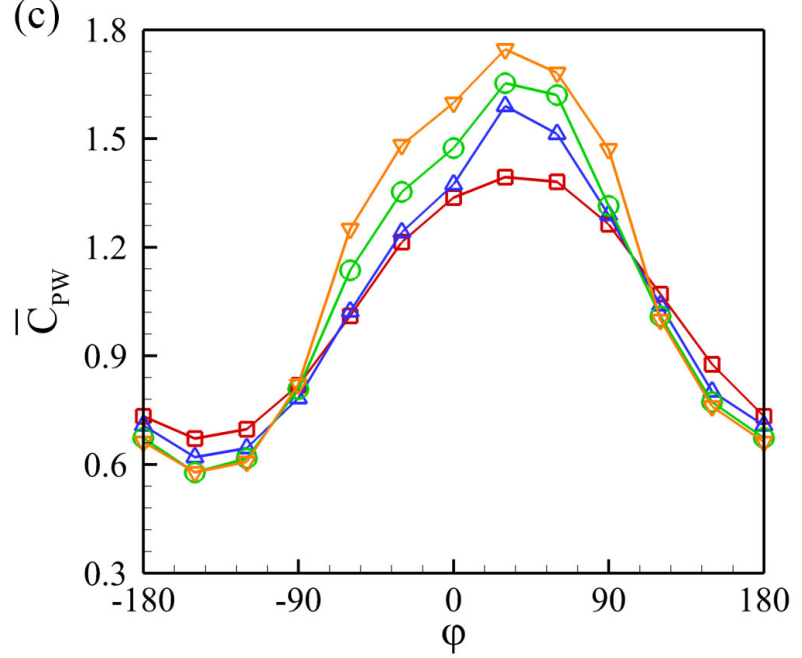

(b)

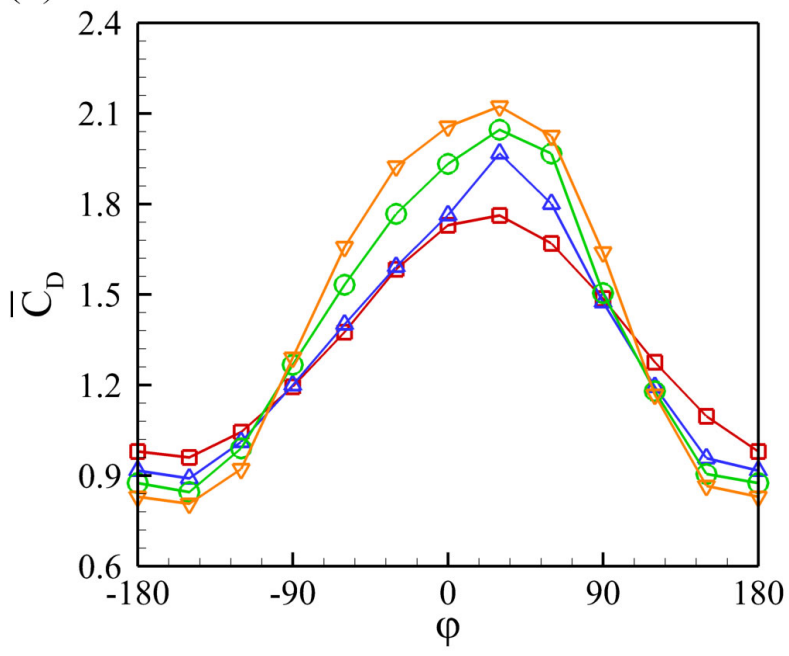

(d)

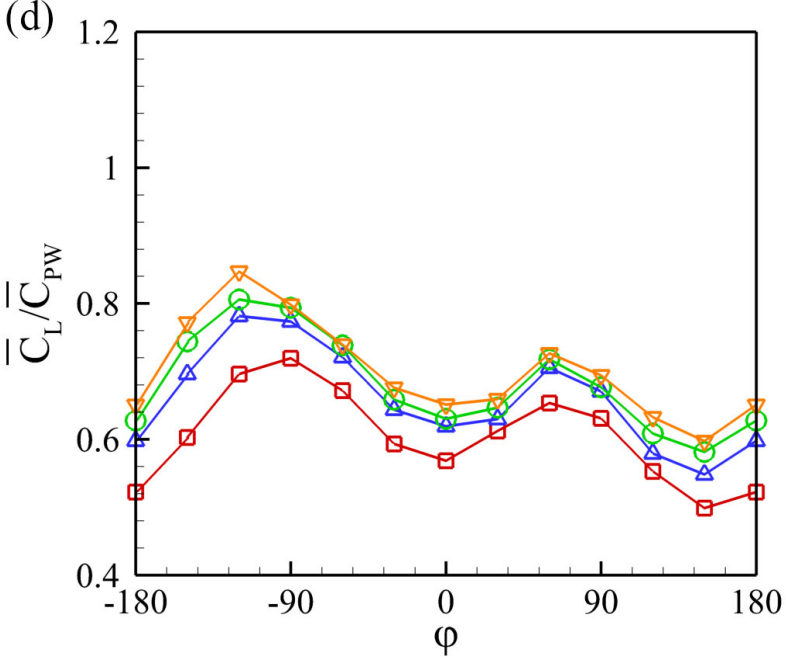

Fig. 15 Aerodynamic performance of plates with various deflection phase difference at $\beta_{T}=60^{\circ}$ for different Reynolds numbers. Cycle-averaged lift (a), drag (b), and power (c) coefficients, and lift-topower ratio (d). 
(a)

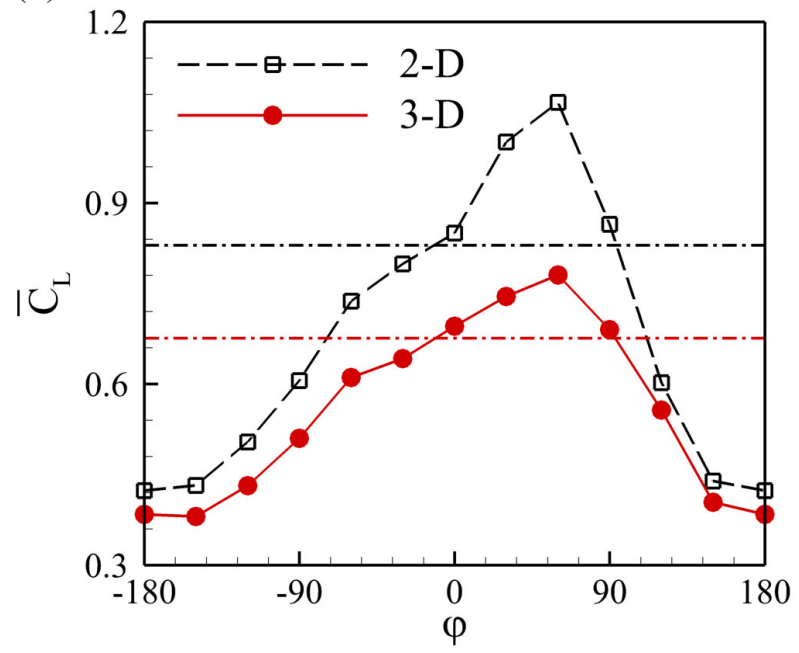

(c)

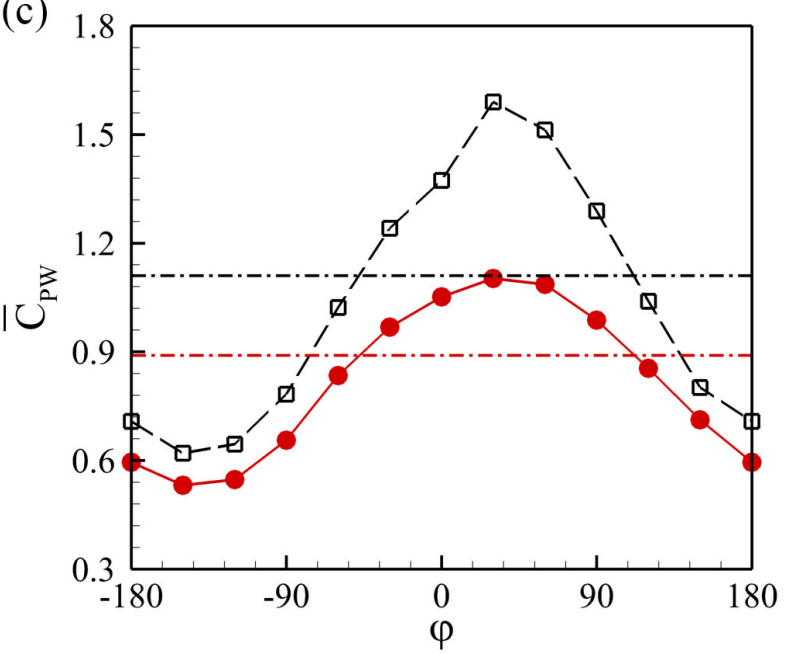

(b)

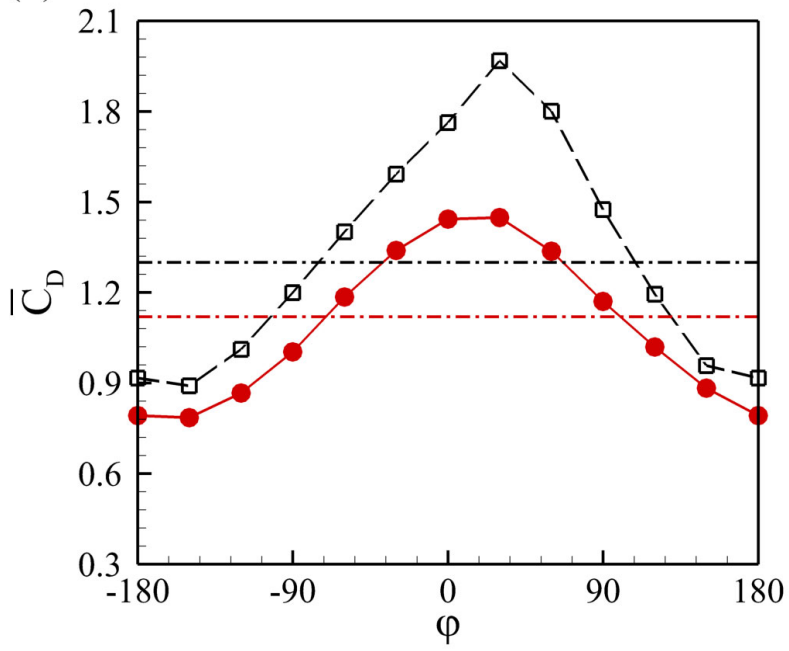

(d)

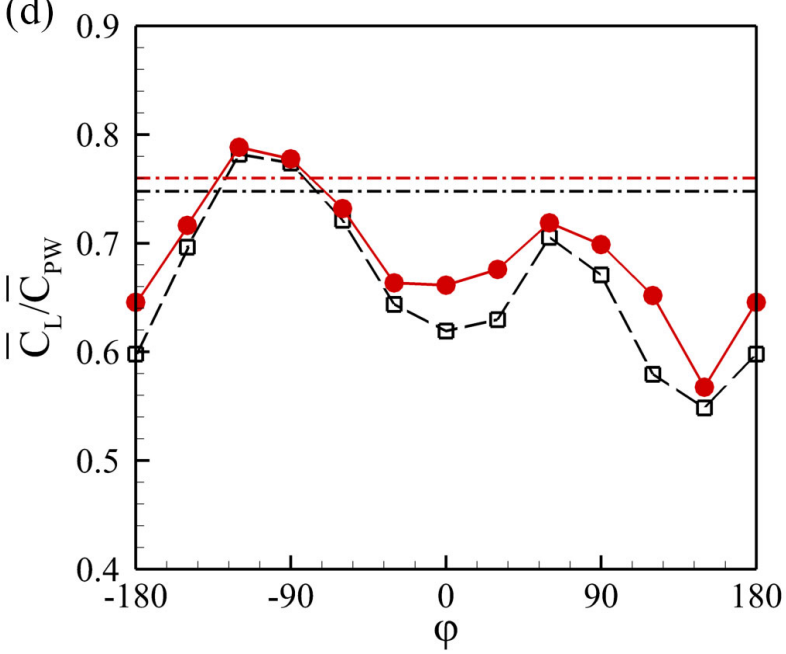

Fig. 16 Comparison of aerodynamic performance between 2-D plate and 3-D plate $(A R=2)$ at $\beta_{T}=60^{\circ}$ for different TEF deflection phase difference. Cycle-averaged lift (a), drag (b), and power (c) coefficients, and lift-to-power ratio (d).

\subsection{Effect of three-dimensionality}

At last, the effects of TEF three-dimensionality on the aerodynamic performance and flow modulation are investigated. Computations are performed using an $A R=2$ plate at $R e=100$ with $H_{L}=0.75 c$, $\beta_{T}=60^{\circ}$ and various $\varphi$ for total four flapping cycles. A notable difference between the 3-D simulation and 2-D simulation is that the 3-D flow quickly reaches a nearly periodic state after approximately two cycles. In the following discussion, the mean forces and power coefficients for 3-D cases are calculated based on the averages of the last two cycles.

Fig. 16 shows the mean force, power coefficients and lift-to-power ratio for 3-D cases at various deflection phase difference. The corresponding 2-D cases are re-plotted for comparison. In general, the overall trend demonstrated by the 3-D cases follows that of the 2-D cases but with smaller amplitude. Based on previous studies, the force fluctuation per unit span is suppressed in 3-D cases due to the 
existence of tip vortices (Taira and Colonius, 2009), which results in a smaller mean force and smaller power coefficients.

(a)

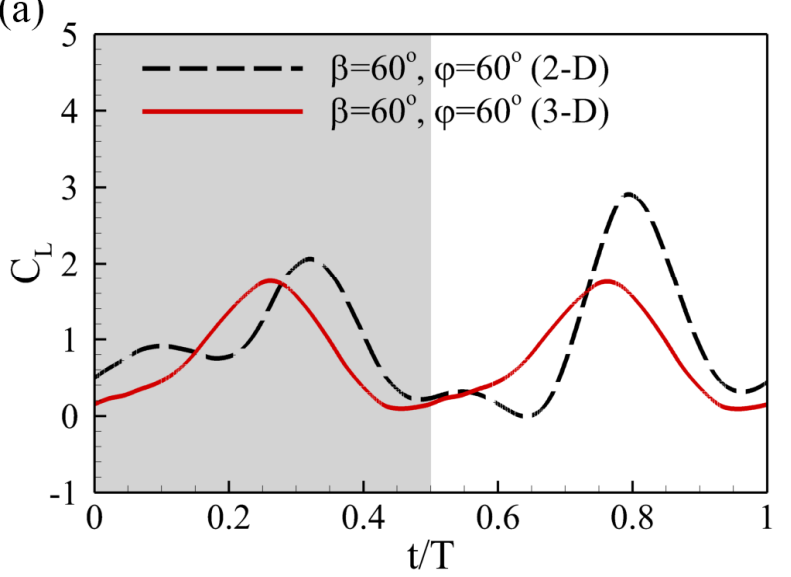

(c)

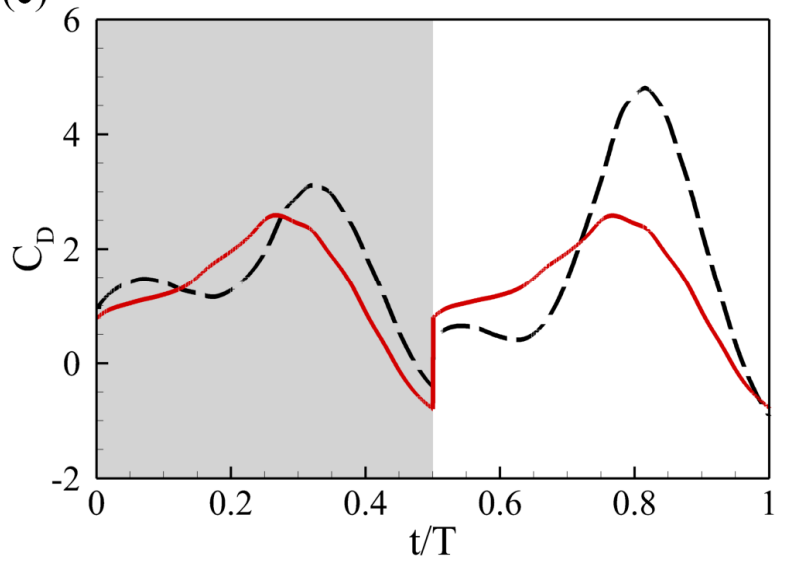

(b)

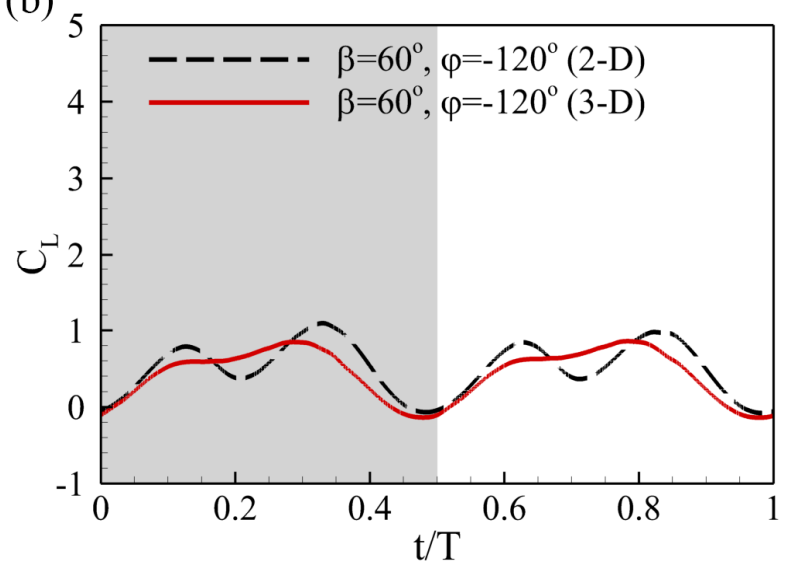

(d)

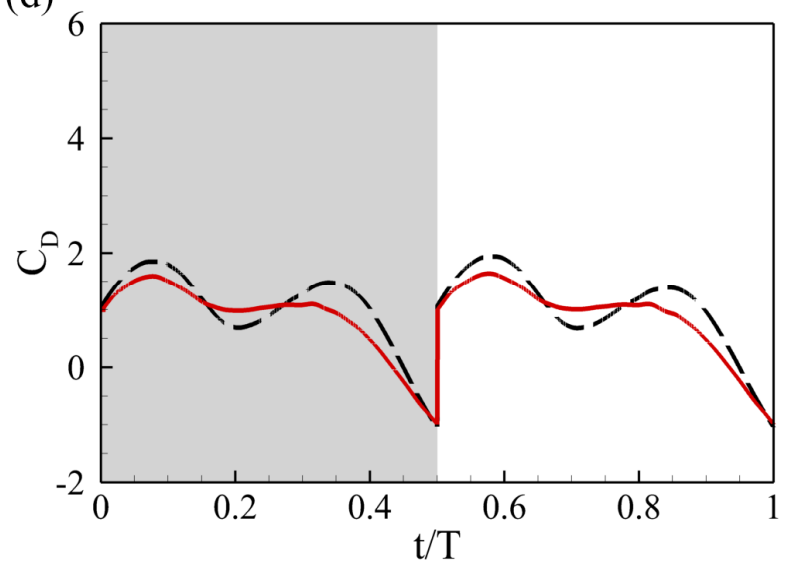

Fig. 17 Comparison of instantaneous lift (a-b) and drag (c-d) coefficients between the 2-D and 3-D cases for positive and negative camber formations.

The time histories of force coefficients at $\beta_{T}=60^{\circ}$ and $\varphi=60^{\circ},-120^{\circ}$ are plotted in Fig. 17 to help us better illustrate the difference between 3-D and 2-D cases. First, the double peaks of each half-stroke for the 2-D cases merge into a single peak in the 3-D cases. In addition, the force production for each downand up- stroke is comparable in 3-D cases. A possible reason is that due to the absence of the span-wise flow and tip vortices, the 2-D case is much more sensitive to the surrounding flow field and vortex interactions than its 3-D case is. Moreover, in 3-D, the tip vortex and span-wise flow stabilizes the LEV and prevents it from shedding (Ellington et al., 1996). 

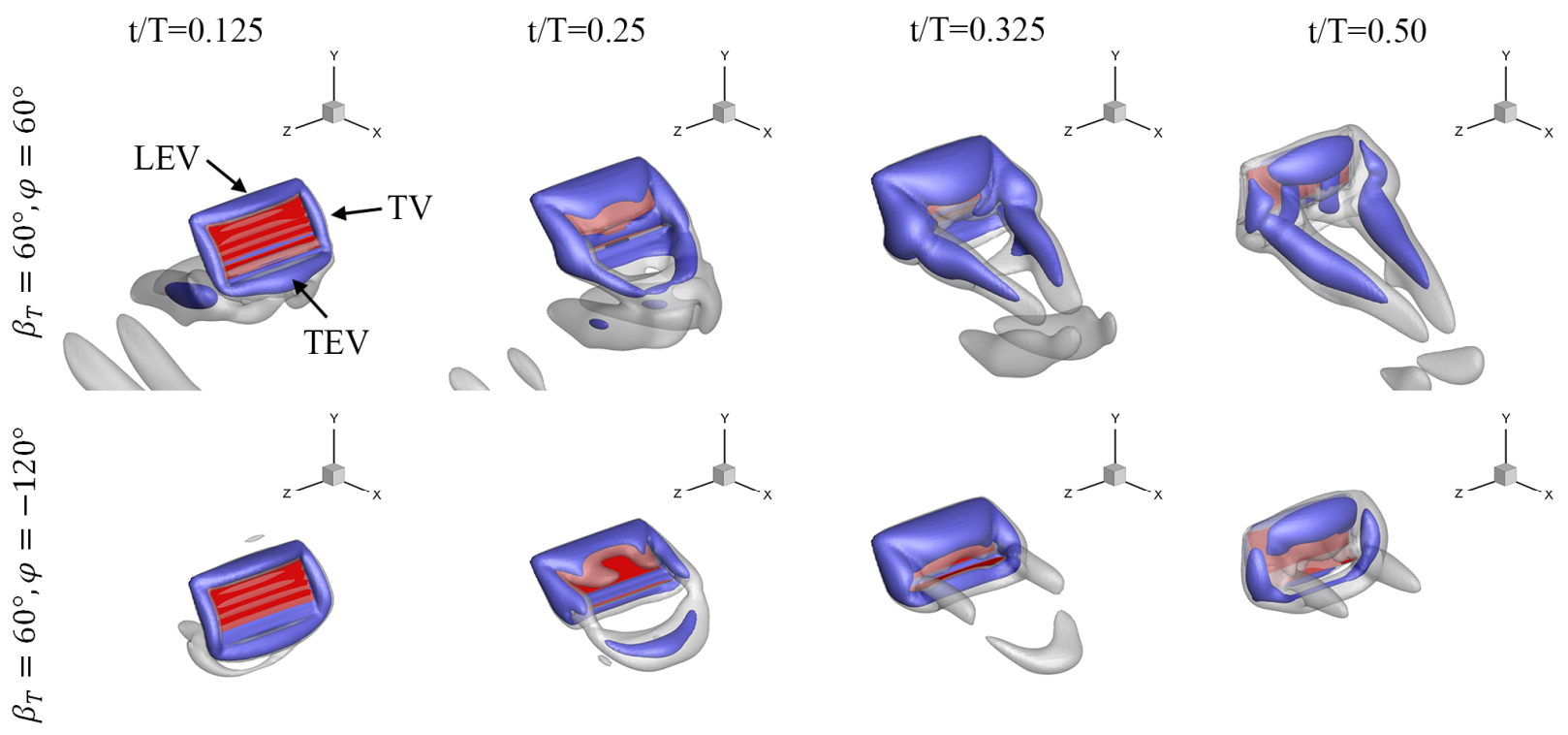

Fig. 18 Comparison of vortical structures between positive $\left(\beta_{T}=60^{\circ}, \varphi=60^{\circ}\right)$ and negative $\left(\beta_{T}=60^{\circ}, \varphi=-120^{\circ}\right)$ camber formations at selected instants during the down-stroke of the $4^{\text {th }}$ flapping cycle.

Fig. 18 shows 3-D wake structures obtained using the iso-surface of Q-criteria (Hunt et al., 1988) at selected instants for both positive and negative camber cases. Two iso-surfaces are plotted to highlight the inner core $(\mathrm{Q}=3.0)$ and outer shell $(\mathrm{Q}=1.0)$ of the vortex structure. The corresponding instantaneous force histories are presented in Fig. 17. Because both the force history and vortex formation are symmetric during the down- and up-strokes, only the down-stroke vortex formation is presented here. In general, the flow structures is similar as reported in previous literature (Shyy et al., 2009). At the beginning of the down-stroke, the flow separates from the leading edge and tips, which act as strong sources of vorticity. Vorticity is gradually fed into the surrounding flow field, and forms the leading-edge, trailing-edge and tip vortices as vortex sheets rolling up from each edge. As the leading-edge vortex (LEV) grows, the trailing-edge vortex (TEV) and symmetric tip vortices (TVs) together form a vortex ring at the bottom of the plate. With the growth of the vortex structure, the plate gradually reaches the peak lift. During the second half of the down-stroke, the LEV vortex starts to detach from the suction side of the plate due to the continuous increase of the plate pitching angle. As a consequence, the force starts to drop and gradually reaches its minimum value. The comparison of the vortex structure between positive and negative cases reveals that the positive camber case $\left(\beta_{T}=60^{\circ}, \varphi=60^{\circ}\right)$ forms a larger LEV with relatively stronger TVs than the negative camber case $\left(\beta_{T}=60^{\circ}, \varphi=-120^{\circ}\right)$. The same phenomenon can be observed from the slice cuts of the vorticity field.

In Fig. 19, the slice cuts are taken at the tip, a distance of $0.5 \mathrm{c}$ from the tip, and at mid-span during the down-stroke. As in the 2-D cases, the LEV of the positive camber case shows better LEV attachment than that in the negative camber case, as indicated by the snapshots captured at $t / T=0.25$. During the translation process, the positive camber formation enhances the tip vortex and creates a stronger LEV. Furthermore, a double core vortex (at $\mathrm{t} / \mathrm{T}=0.325$ ) is formed at the tip when a positive camber is formed, which might be because of the enhancement of the tip vortex. It is also observed that the shed vortices are more dissipative in the 3-D case than in the 2-D case by comparing the vorticity plots in the far wake (Fig. 9). 

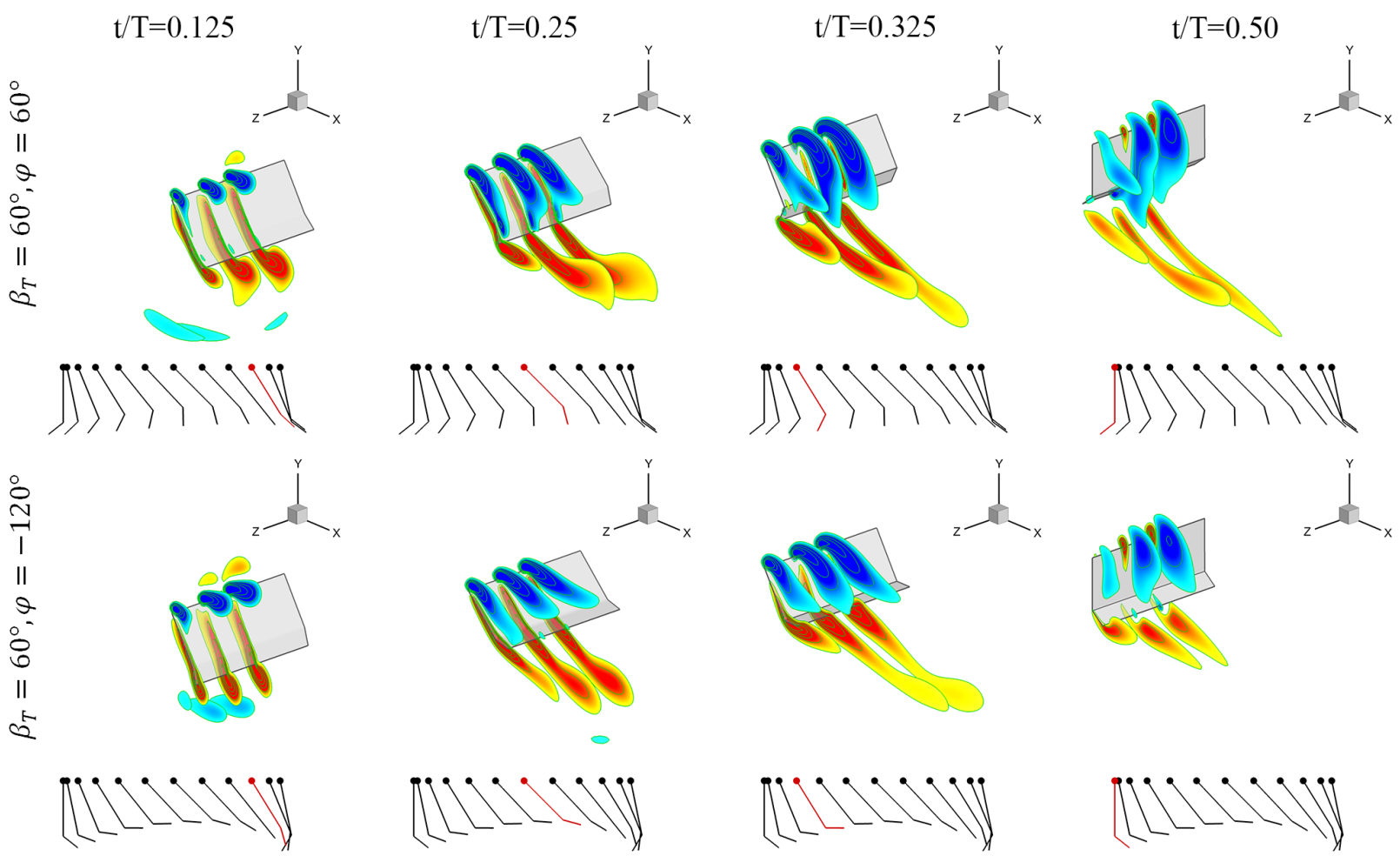

Fig. 19 Comparison of Z-vorticity contours between positive $\left(\beta_{T}=60^{\circ}, \varphi=60^{\circ}\right)$ and negative $\left(\beta_{T}=60^{\circ}, \varphi=-120^{\circ}\right.$ ) camber formation at selected time instants during the down-stroke of the $4^{\text {th }}$ flapping cycle. The slices cuts are taken at plate tip, at a distance of $0.5 c$ from the tip, and at the midspan.

\section{Conclusions}

The effects of the dynamic trailing-edge flap (TEF) on the aerodynamic performance and flow structures of both 2-D and 3-D flapping plates has been numerically studied for Reynolds number range of 50 to 400. Various parameters for controlling TEF kinematics, such as deflection phase difference, deflection amplitude, and hinge location are considered. Our results suggest that the TEF deflection phase difference play a more dominant role than other parameters. The lift enhancement always happens for positive camber formation cases (i.e., deflection phase difference $\left.\varphi \in\left(-90^{\circ}, 90^{\circ}\right)\right)$. By tailoring the deflection phase difference and deflection amplitude, the lift production can be increased up to $26 \%$ comparing to a fully rigid plate. The analysis of the associated unsteady flow structures has shown that the dynamic motion of TEF is able to feed extra circulation into the trailing-edge vortex which induces a stronger counterpart leading-edge vortex. As a result of local flow modulation by TEF, the vortex on the suction side is pulled down closer to the plate (stall delay), which leads to the improvement of lift production. Our results also suggest that the plate performance is insensitive to the hinge location when the effective pitching angle kept the same. Changing Reynolds numbers only affects the magnitude of mean force coefficients whereas the overall trend of TEF effect maintains the same. Results from the lowaspect-ratio plates indicate that the general conclusion drawn from $2 \mathrm{D}$ cases is applicable to $3 \mathrm{D}$ cases even though the flow features are different at certain levels. 


\section{Acknowledgements}

This work is supported under AFOSR FA9550-11-1-0058 and AFOSR FA9550-12-1-007 monitored by Dr. Douglas Smith and NSF CEBT-1313217.

\section{References}

Bergou, A.J., Ristroph, L., Guckenheimer, J., Cohen, I., Wang, Z.J., 2010. Fruit Flies Modulate Passive Wing Pitching to Generate In-Flight Turns. Physical Review Letters 104.

Brian C. Prock, T.A.W., and William A. Crossley, 2002. Morphing Airfoil Shape Change Optimization with Minimum Actuator Energy as an Objective, 9th AIAA/ISSMO Symposium on Multidisciplinary Analysis and Optimization, Atlanta, Georgia.

Dai, H., Luo, H., Doyle, J.F., 2012. Dynamic pitching of an elastic rectangular wing in hovering motion. Journal of Fluid Mechanics 693, 473-499.

Dickinson, M.H., Lehmann, F.O., Sane, S.P., 1999. Wing rotation and the aerodynamic basis of insect flight. Science 284, 1954-1960.

Dong, H., Mittal, R., Najjar, F.M., 2006. Wake topology and hydrodynamic performance of low-aspectratio flapping foils. Journal of Fluid Mechanics 566, 309-343.

Du, G., Sun, M., 2008. Effects of unsteady deformation of flapping wing on its aerodynamic forces. Appl. Math. Mech.-Engl. Ed. 29, 731-743.

Eldredge, J.D., 2007. Numerical simulation of the fluid dynamics of 2D rigid body motion with the vortex particle method. Journal of Computational Physics 221, 626-648.

Eldredge, J.D., Toomey, J., Medina, A., 2010. On the roles of chord-wise flexibility in a flapping wing with hovering kinematics. Journal of Fluid Mechanics 659, 94-115.

Ellington, C.P., vandenBerg, C., Willmott, A.P., Thomas, A.L.R., 1996. Leading-edge vortices in insect flight. Nature 384, 626-630.

Gerontakos, P., Lee, T., 2008. PIV study of flow around unsteady airfoil with dynamic trailing-edge flap deflection. Experiments in fluids 45, 955-972.

Ho, C.M., Tai, Y.C., 1996. Review: MEMS and its applications for flow control. Journal of Fluids Engineering-Transactions of the Asme 118, 437-447.

Hunt, J.C.R., Wray, A.A., Moin, P., 1988. Eddies, streams, and convergence zones in turbulent flows. Center for Turbulence Research Report CTR-S88.

Kang, C.K., Aono, H., Cesnik, C.E.S., Shyy, W., 2011. Effects of flexibility on the aerodynamic performance of flapping wings. Journal of Fluid Mechanics 689, 32-74.

Koehler, C., Liang, Z.X., Gaston, Z., Wan, H., Dong, H.B., 2012. 3D reconstruction and analysis of wing deformation in free-flying dragonflies. Journal of Experimental Biology 215, 3018-3027.

Le, T.Q., Van Truong, T., Park, S.H., Truong, T.Q., Ko, J.H., Cheol, H., 2013. Improvement of the aerodynamic performance by wing.

Lee, T., Gerontakos, P., 2006. Dynamic stall flow control via a trailing-edge flap. AIAA journal 44, 469480.

Lee, T., Su, Y., 2011a. Unsteady airfoil with a harmonically deflected trailing-edge flap. Journal of Fluids and Structures 27, 1411-1424.

Lee, T., Su, Y.Y., 2011b. Lift enhancement and flow structure of airfoil with joint trailing-edge flap and Gurney flap. Experiments in Fluids 50, 1671-1684.

Li, C., Dong, H., Ren, Y., 2014. A Numerical Study of Flapping Plates Hinged with a Trailing-Edge Flap, 32nd AIAA Applied Aerodynamics Conference. AIAA, Atlanta, GA.

Liu, Y., Cheng, B., Deng, X., 2013. An experimental Study of Dynamic Trailing Edge Deflections on a Two Dimensional Translating Wing, 31st AIAA Applied Aerodynamics Conference. AIAA. 
Mittal, R., Dong, H., Bozkurttas, M., Najjar, F.M., Vargas, A., von Loebbecke, A., 2008. A versatile sharp interface immersed boundary method for incompressible flows with complex boundaries. Journal of Computational Physics 227, 4825-4852.

Shoele, K., Zhu, Q., 2013. Performance of a wing with nonuniform flexibility in hovering flight. Physics of Fluids 25.

Shyy, W., Aono, H., Chimakurthi, S.K., Trizila, P., Kang, C.K., Cesnik, C.E.S., Liu, H., 2010. Recent progress in flapping wing aerodynamics and aeroelasticity. Progress in Aerospace Sciences 46, 284-327.

Shyy, W., Lian, Y., Tang, J., Liu, H., Trizila, P., Stanford, B., Bernal, L., Cesnik, C., Friedmann, P., Ifju, P., 2008. Computational aerodynamics of low Reynolds number plunging, pitching and flexible wings for MAV applications. Acta Mechanica Sinica 24, 351-373.

Shyy, W., Liang, Y., Tang, J., Viieru, D., Liu, H., 2011. Aerodynamics of Low Reynolds Number Flyers. Cambridge University Press.

Shyy, W., Trizila, P., Kang, C.K., Aono, H., 2009. Can Tip Vortices Enhance Lift of a Flapping Wing? Aiaa Journal 47, 289-293.

Sun, M., Tang, H., 2002. Unsteady aerodynamic force generation by a model fruit fly wing in flapping motion. Journal of Experimental Biology 205, 55-70.

Taira, K., Colonius, T.I.M., 2009. Three-dimensional flows around low-aspect-ratio flat-plate wings at low Reynolds numbers. Journal of Fluid Mechanics 623, 187-207.

Toomey, J., Eldredge, J.D., 2008. Numerical and experimental study of the fluid dynamics of a flapping wing with low order flexibility. Physics of Fluids 20.

Trizila, P., Kang, C.K., Aono, H., Shyy, W., Visbal, M., 2011. Low-Reynolds-Number Aerodynamics of a Flapping Rigid Flat Plate. AIAA Journal 49, 806-823.

Valasek, J., 2012. Morphing aerospace vehicles and structures. Wiley, Chichester.

Vanella, M., Fitzgerald, T., Preidikman, S., Balaras, E., Balachandran, B., 2009. Influence of flexibility on the aerodynamic performance of a hovering wing. Journal of Experimental Biology 212, 95-105.

Walker, S.M., Thomas, A.L.R., Taylor, G.K., 2009. Deformable wing kinematics in the desert locust: how and why do camber, twist and topography vary through the stroke? Journal of the Royal Society Interface 6, 735-747.

Walker, S.M., Thomas, A.L.R., Taylor, G.K., 2010. Deformable wing kinematics in free-flying hoverflies. Journal of the Royal Society Interface 7, 131-142.

Wan, H., Dong, H., Huang, G.P., 2012. Hovering Hinge-Connected Flapping Plate with Passive Deflection. AIAA Journal 50, 2020-2027.

Wang, H., Zeng, L.J., Liu, H., Yin, C.Y., 2003. Measuring wing kinematics, flight trajectory and body attitude during forward flight and turning maneuvers in dragonflies. Journal of Experimental Biology 206, 745-757.

Wang, S., Zhang, X., He, G., Liu, T., 2014. Lift enhancement by dynamically changing wingspan in forward flapping flight. Physics of Fluids 26, 061903.

Wang, Z.J., Birch, J.M., Dickinson, M.H., 2004. Unsteady forces and flows in low Reynolds number hovering flight: two-dimensional computations vs robotic wing experiments. Journal of Experimental Biology 207, 449-460.

Xu, M., Wei, M., Li, C., Dong, H., 2015. Adjoint-based optimization of flapping plates hinged with a trailing-edge flap. Theoretical and Applied Mechanics Letters 5, 1-4.

Yin, B., Luo, H.X., 2010. Effect of wing inertia on hovering performance of flexible flapping wings. Physics of Fluids 22.

Zhang, G., Sun, J., Chen, D., Wang, Y., 2008. Flapping motion measurement of honeybee bilateral wings using four virtual structured-light sensors. Sensors and Actuators A: Physical 148, 19-27.

Zhao, L., Deng, X.Y., Sane, S.P., 2011. Modulation of leading edge vorticity and aerodynamic forces in flexible flapping wings. Bioinspir. Biomim. 6.

Zhao, L., Huang, Q.F., Deng, X.Y., Sane, S.P., 2010. Aerodynamic effects of flexibility in flapping wings. Journal of The Royal Society Interface 7, 485-497. 
1 Zheng, L., Hedrick, T.L., Mittal, R., 2013. Time-varying wing-twist improves aerodynamic efficiency of 2 forward flight in butterflies. PloS one 8, e53060. 